\title{
A NEURAL NETWORK MODEL OF BACKWARD PRIMING
}

\author{
A Thesis \\ Presented to \\ The Faculty of Graduate Studies \\ of \\ The University of Guelph
}

by

JEFFREY FRANSON

In partial fulfilment of requirements

for the degree of

Master of Arts

April, 2008

(C) Jeffrey Franson, 2008 


$\begin{array}{ll}\begin{array}{l}\text { Library and } \\ \text { Archives Canada }\end{array} & \begin{array}{l}\text { Bibliothèque et } \\ \text { Archives Canada }\end{array} \\ \begin{array}{l}\text { Published Heritage } \\ \text { Branch }\end{array} & \begin{array}{l}\text { Direction du } \\ \text { Patrimoine de l'édition }\end{array} \\ \begin{array}{l}\text { 395 Wellington Street } \\ \text { Ottawa ON K1A 0N4 } \\ \text { Canada }\end{array} & \begin{array}{l}\text { O95, rue Wellington } \\ \text { Ottawa ON K1A 0N4 } \\ \text { Canada }\end{array}\end{array}$

Your file Votre référence ISBN: 978-0-494-42780-4

Our file Notre référence

ISBN: 978-0-494-42780-4

NOTICE:

The author has granted a nonexclusive license allowing Library and Archives Canada to reproduce, publish, archive, preserve, conserve, communicate to the public by telecommunication or on the Internet, loan, distribute and sell theses worldwide, for commercial or noncommercial purposes, in microform, paper, electronic and/or any other formats.

The author retains copyright ownership and moral rights in this thesis. Neither the thesis nor substantial extracts from it may be printed or otherwise reproduced without the author's permission.
AVIS:

L'auteur a accordé une licence non exclusive permettant à la Bibliothèque et Archives Canada de reproduire, publier, archiver, sauvegarder, conserver, transmettre au public par télécommunication ou par l'Internet, prêter, distribuer et vendre des thèses partout dans le monde, à des fins commerciales ou autres, sur support microforme, papier, électronique et/ou autres formats.

L'auteur conserve la propriété du droit d'auteur et des droits moraux qui protège cette thèse. $\mathrm{Ni}$ la thèse ni des extraits substantiels de celle-ci ne doivent être imprimés ou autrement reproduits sans son autorisation.
In compliance with the Canadian

Privacy Act some supporting forms may have been removed from this thesis.

While these forms may be included in the document page count, their removal does not represent any loss of content from the thesis.
Conformément à la loi canadienne sur la protection de la vie privée, quelques formulaires secondaires ont été enlevés de cette thèse.

Bien que ces formulaires aient inclus dans la pagination, il n'y aura aucun contenu manquant.

\section{Canadä}




\begin{abstract}
A NEURAL NETWORK MODEL OF BACKWARD PRIMING

Jeffrey Franson

University of Guelph, 2008

Advisor: H. Marmurek

Semantic priming refers to facilitation in the recognition or naming of a word (the target, e.g., KING) following a semantically related word (the prime, e.g. CROWN). Backward priming demonstrates similar effects for related words presented in the reverse order. The first goal of this study was to examine priming effects in both directions for asymmetrically associated words. It also investigated the contributions of automatic and strategic processing mechanisms, lexical frequency, semantic relatedness, and association strength to priming in a lexical decision task. The second goal was to develop a neural network model capable of simulating those priming effects. Results showed that both the human participants and the neural network model displayed both forward and backward priming effects. The results of the human participants and model were then compared in order to evaluate the neural network as a model of human lexical processing.
\end{abstract}




\section{ACKNOWLEDGEMENTS}

I would like to thank my advisor, Dr. Harvey Marmurek, for all his support and guidance throughout the process of writing and defending my thesis. I would also like to extend my gratitude towards Dr. Stefan Kremer of the Computing and Information Science department for his advice and especially his help with the neural network portion of my thesis. I am also grateful for the NACS graduate students in my cohort who provided helpful advice and much needed encouragement along the way. Finally, I would like to say thank you to members of my family. In particular, my parents, Del and Angela Franson, who have long supported me during my university education and my aunt, Francesca Mastrella, who provided me a home away from home while I completed my Masters degree at the University of Guelph. 


\section{TABLE OF CONTENTS}

Acknowledgements $\quad$ i

Table of Contents $\quad$ ii

List of Tables $\quad$ iv

List of Figures vii

Introduction 1

Literature Review of Backward Priming 3

$\begin{array}{ll}\text { Literature Review of Neural Networks } & 11\end{array}$

Neural Network Models of Semantic Priming 13

$\begin{array}{ll}\text { Current Study } & 15\end{array}$

$\begin{array}{ll}\text { Predictions for the Experiment } & 19\end{array}$

$\begin{array}{lr}\text { Predictions for the Neural Network Model } & 20\end{array}$

$\begin{array}{ll}\text { The Experiment } & 23\end{array}$

Method

$\begin{array}{ll}\text { Participants } & 23\end{array}$

$\begin{array}{ll}\text { Stimuli } & 23\end{array}$

$\begin{array}{ll}\text { Materials } & 25\end{array}$

$\begin{array}{ll}\text { Procedure } & 25\end{array}$

$\begin{array}{ll}\text { Experimental Results } & 26\end{array}$

$\begin{array}{ll}\text { Discussion } & 33\end{array}$

The Neural Network Model $\quad 38$

Method

$\begin{array}{ll}\text { Neural Network Architecture } & 39\end{array}$ 
$\begin{array}{ll}\text { Stimuli } & 40\end{array}$

$\begin{array}{ll}\text { Training Procedure } & 42\end{array}$

$\begin{array}{ll}\text { Testing Procedure } & 45\end{array}$

Neural Network Results $\quad 46$

$\begin{array}{ll}\text { Discussion } & 49\end{array}$

$\begin{array}{ll}\text { General Discussion } & 57\end{array}$

$\begin{array}{ll}\text { References } & 63\end{array}$

$\begin{array}{ll}\text { Tables } & 72\end{array}$

$\begin{array}{lr}\text { Figures } & 98\end{array}$ 


\section{LIST OF TABLES}

1 Forward and Backward Priming Results (In Milliseconds) From Previous Lexical Decision Task Studies Using Asymmetrically Associated Words

2 Forward and Backward Priming Results (In Milliseconds) From Previous Lexical Decision Task Studies Using Compound Cue Words

3 The Set of Asymmetrically Related Word Pairs Used In The Study With Corresponding Association Strength And Lexical Frequency Values

4 Example of a Base Set With The Eight Possible Experimental Conditions For The Related Word Pair "KING $\rightarrow$ CROWN"

5 Mean Reaction Times and Priming Results From the Experiment

6 Overall Percentage of Errors Made In Each Priming Condition

7 Analysis of Variance for Participant Mean Decision Latencies

$8 \quad$ Analysis of Variance for Word Item Mean Decision Latencies

9 Analysis of Variance for Participant Mean Error Percentages

10 Analysis of Variance for Word Item Mean Error Percentages

11 Bivariate and Partial Correlations of the Predictors with Forward Priming

12 Summary of the Multiple Regression Analysis for Variables Predicting the Magnitude of Forward Priming

13 Bivariate and Partial Correlations of the Predictors with Backward Priming

14 Summary of the Multiple Regression Analysis for Variables Predicting the Magnitude of Backward Priming

15 Bivariate and Partial Correlations of the Predictors with Forward Priming Using the Subset of Related Word Pairs 
16 Summary of the Multiple Regression Analysis for Variables Predicting the Magnitude of Forward Priming Using the Subset of Related Word Pairs

17 Bivariate and Partial Correlations of the Predictors with Backward Priming Using the Subset of Related Word Pairs

18 Bivariate and Partial Correlations of the Predictors with Backward Priming Using the Subset of Related Word Pairs

19 Mean Error Scores (Root MSE) and Priming Results For The Neural Network

20 Analysis of Variance for Neural Network Simulation (Participant) Mean Root MSE Values

21 Analysis of Variance for Neural Network Word Item Mean Root MSE Values

22 Bivariate and Partial Correlations of the Predictors with Forward Priming in the Neural Network

23 Summary of the Multiple Regression Analysis for Lexical Frequency of the Target Word and Forward Association Predicting Magnitude of Forward Priming in the Neural Network

24 Bivariate and Partial Correlations of the Predictors with Backward Priming in the Neural Network

25 Summary of the Multiple Regression Analysis for Lexical Frequency of the Target Word and Forward Association Predicting Magnitude of Backward Priming in the Neural Network 


\section{LiST OF Figures}

1 Architecture Of The Simple Recurrent Neural Network Used In The Study

2 Cluster Analysis For Hidden Activation States Of Related Prime And Target

Words When Presented In Isolation and Distinguished By Semantic

Relatedness

3 Cluster Analysis For Hidden Activation States For Unrelated Prime And Target

Words When Presented In Isolation Distinguished By Semantic

Relatedness

4 Cluster Analysis For Context Layer Activation States For Related Prime And

Target Words When Presented Together In the Backward Direction

Distinguished By Semantic Relatedness 
Semantic priming refers to facilitation in the recognition or naming of a word (the target) following a semantically related word (the prime). Psychologists have studied this phenomenon extensively over the last few decades in both visual and auditory tasks involving word naming, lexical decision, and semantic categorisation. The pervasive effects of semantic priming found in this research have provided a better understanding of how the cognitive system stores and processes lexical information.

Two types of semantic relations contribute to semantic priming: purely semantic relations, and associative relations. Purely semantic relations involve priming effects that occur between words that share similar semantic features or belong to the same semantic category (e.g., BREAD-CAKE). An associative relation between two words is identified via free association reflecting how likely one word is brought to mind when the other is presented. Associative relations are considered directional in nature. Thus, the strength of an association between associated words depends on their order of presentation. Most associatively related words have a semantic relation as well, but like purely semantically related words, the nature of the semantic relation can vary (e.g., functional:

HAMMER $\rightarrow$ NAIL; category coordinates: LIZARD $\rightarrow$ SNAKE; script relations: $\mathrm{ONION} \rightarrow \mathrm{CRY}$ ).

In general, semantically related words that also have an associative relation produce stronger priming effects (called an "associative boost") than purely semantically related words (Hutchinson, 2003; Lucas, 2000; Moss, Ostrin, Tyler, \& Marlen-Wilson, 1995). For associatively/semantically related words, there have been attempts to delineate the respective contributions of the associative and semantic relations towards priming (e.g., Chiarello, Burgess, Richards, \& Pollock, 1990; Schreuder, Flores d' 
Arcais, \& Glazenburg, 1984; Shelton \& Martin, 1992). The most conclusive study on the matter demonstrated that associatively related words that have a minimal semantic relation produce less priming than both normal associatively/semantically related words and purely semantically related words that are category coordinates or synonyms (Mass et al., 1995).

Priming effects between purely semantically related items can be explained as a result of the number of overlapping semantic features shared between related items (MacNamara, 2005). The overlapping features activated when the prime word is presented remain activated when the target word is presented and, thus, facilitate target word recognition. For associatively related words, it is still unclear how the associative relation produces priming effects. McRae and Boisvert (1998) have argued that, compared to a purely semantic relation, an associative relation is simply a stronger semantic relationship. Other researchers have argued that associative relations are independent of semantic relations and arise from the frequent lexical co-occurrence of associatively related word found within language use (Fischler, 1977; Fodor, 1983). Durgunoglu and Nelly (1987) found evidence that associative relations are produced from the combined influence of the frequency of lexical co-occurrence and the semantic relation existing between associatively related words. In their study, the researchers had participants learn a set of novel word pairs and then had them read textual corpora with these word pairs frequently embedded within the same sentence contexts. They found that priming effects were found only in novel word pairs that had been provided with a meaningful relationship between them during the learning phase. 
Priming effects between purely semantically related words tend to be symmetrical in terms of the order of presentation of the word pair (Thompson-Schill, Kurtz, \& Gabrieli, 1998). On the other hand, priming effects for associatively related words are often asymmetric depending on the order of word presentation (Neely, 1991). For example, CROWN $\rightarrow$ KING typically elicits more priming than KING $\rightarrow$ CROWN. This asymmetry can be attributed to a stronger association existing between the related words in the priming direction that elicits greater priming effects. Priming that arises in the weaker association presentation direction is known as backward priming.

\section{Literature Review of Backward Priming}

In backward priming studies, related words have a stronger associative relation in the forward priming direction than in the backward direction (e.g., CROWN elicits the word KING more often than KING elicits the word CROWN). Nevertheless, reliable backward priming effects have been found in a number of lexical decision and word naming studies (Chwilla, Hagoort, \& Brown, 1998; Hutchinson, 2002; Kahan, Neely, \& Forsythe, 1998; Koriat, 1981; Peterson \& Simpson, 1989; Seidenberg, Waters, Snaders, \& Langer, 1984; Shelton \& Martin, 1992; and Thompson-Schill, Kurtz, \& Gabrieli, 1998). There are two types of related word pairs that have been used in backward priming studies. Asymmetrically associated words (e.g., CROWN $\rightarrow$ KING) are considered to share both a semantic and associative relationship. Compound cues (e.g., $\mathrm{COAT} \rightarrow \mathrm{RACK})$, on the other hand, are considered to be only associative in nature since the prime and target essentially form a compound word. In the first word recognition study of backward priming, Koriat (1981) found equal amounts of priming in both directions using asymmetrical associates. Other results have varied in terms of the 
relative strength of forward and backward priming. Table 1 presents the six backward priming studies that used asymmetrically associated words (Chwilla, Hagoort, \& Brown, 1998; Hutchinson, 2002; Kahan, Neely, \& Forsythe, 1998; Koriat, 1981; Peterson \& Simpson, 1989; Thompson-Schill, Kurtz, \& Gabrieli, 1998).

Table 1 suggests the variation may arise from differing experimental conditions across these studies. Overall, forward priming tends to be moderately stronger $(M=37$ $\mathrm{ms}$ ) than backward priming ( $M=23 \mathrm{~ms})$. It is important to note, though, that these effects are averaged across studies that have tested different stimulus sets. The Kahan et al. (1999) and Peterson and Simpson (1989) studies used identical sets of asymmetrically associated words, but researchers did not distinguish asymmetric associates from compound cues. Thompson-Schill et al. (1998) used most of the same asymmetric associates as Kahan et al., but the researchers also included some of their own asymmetrically associated word pairs. Thompson-Schill et al. also based their selection of asymmetrically associated word pairs based on an association strength measure they devised. On the other hand, Kahan et al. (1998) used Nelson, McEvoy, and Schreiber's (1998) more established University of South Florida Free Association Norms to select their asymmetrically related word set. Hutchinson's (2000) word pairs were a mixture of items taken from Kahan et al. and Thompson-Schill et al., but their selection criterion was based on the University of South Florida Free Association Norms. Chwilla et al. (1998) tested a set of asymmetric associates that was smaller than Hutchinson's and was selected using association strengths from a Dutch database of word association norms.

The main methodological inconsistencies across studies evident in Table 1 are the differing SOAs or stimulus onset asynchronies (the time interval between the 
presentation onset of the prime word and the onset presentation of the target word), the relatedness proportion (RP), and non-word ratio (NR). The relatedness proportion refers to the proportion of related word pairs to unrelated word pairs; the non-word ratio is the proportion of trials that involve non-word target items following a prime word relative to trials with word target items. Both of these variables are important because researchers use them as a means of determining the cognitive mechanisms involved in semantic priming.

Two general types of processing may contribute to semantic priming: automatic versus strategic. Automatic processing is believed to occur in tasks that require very rapid information processing, little conscious awareness, and minimal attention. Strategic processing tasks involve slower information processing, require greater attention, and are under a greater degree of conscious control. Most researchers have contended that automatic processing mechanisms rely upon associative and semantic features between related words to facilitate target word recognition (Hutchinson, 2003; Lucas, 2000; MacNamara, 2005; Moss et al., 1995; Perea \& Gotor, 1997; Shelton \& Martin, 1992). In contrast, Thompson-Schill et al. (1998) have argued that only the semantic relationship is involved during automatic processing. Expectancy and semantic matching effects reflect strategic processing mechanisms. Expectancy effects occur when a participant's lexical processing mechanisms activate a set of related target words when a prime word is presented. This can facilitate recognition of a related target word or inhibit unrelated target words. Semantic matching refers to the participant's lexical processing mechanisms search for a semantic relation between a prime and target word. The presence of a semantic relation biases a participant towards a "word" response; in the 
absence of a semantic relation, there is a bias towards a "non-word" response.

In semantic priming tasks, increasing the RP induces expectancy effects and increasing the NR induces semantic matching effects. Increasing the SOA induces the involvement of both effects. As evident in Table 1, most of the previous studies of backward priming involving asymmetrically associated words have either had a long SOA ( $>250 \mathrm{msec})$, a high RP $(>0.50)$, or a high NR $(>0.50)$. Therefore, it is difficult to determine the role of automatic processing in backward priming for asymmetrically associated words.

Table 2 shows the results from backward priming studies using compound cues. On the whole, forward priming ( $M=32 \mathrm{~ms})$ was stronger than backward priming ( $M=$ $22 \mathrm{~ms}$ ). Shelton and Martin (1992) used the same set of compound cues used by Seidenberg, Waters, Sanders, and Langer (1984). Chwilla et al. (1998), Kahan et al. (1999), and Hutchinson (2000) used a larger set of compound cue items than these former two studies but contained most of their compound cues. In contrast to asymmetrically associated words, there is slightly stronger evidence that compound cue words tend to elicit backward priming effects under experimental conditions that are conducive towards strategic priming effects (MacNamara, 2005). Specifically, the studies of compound cues shown in Table 2 have found backward priming effects using longer SOAs and higher RPs than the studies using asymmetrically associated words. ${ }^{1}$ Although Shelton and Martin's first experiment had an RP and NR less than .50, it also had an SOA of 750 milliseconds. In Kahan et al.'s first experiment, priming effects for compound cues were obtained using an SOA of 150 milliseconds, but nevertheless, the researchers used an RP

\footnotetext{
${ }^{1}$ On average, the SOA was $336 \mathrm{~ms}$ for asymmetrically associated words and $481 \mathrm{~ms}$ for compound cues. The average RP was 0.59 for asymmetrically associated words and 0.64 for compound cues. The average NR was 0.71 for both types of related word pairs.
} 
of .80 and a NR of .875 . Therefore, in both of these cases, the experimental conditions were still conducive towards strategic processing.

Further evidence for the involvement of strategic processing in backward priming effects for compound cues is that they lack a semantic relation and usually have very weak or non-existent backward association strength values. On average, compound cues have a lower backward association strength value $(M=0.02)$ than asymmetrically associated words $(M=0.08)(\text { MacNamara, 2005) })^{2}$. As mentioned earlier, both the semantic and associative relations held between a related word pair are believed to be active under automatic processing conditions.

The roles of automatic and strategic processing mechanisms in backward priming for asymmetric associates are less clear. Although asymmetric associates have a semantic relation and a stronger backward association strength than most compound cues, Table 1 also reveals that several studies for asymmetric associates have used a high RP, high NR, and long SOA conditions for their experiment. Because of the shorter SOA and lower RP, Thompson-Schill et al.'s (1998) study is the closest approximation of conditions conducive to automatic processing mechanisms. Thompson-Schill et al. tested forward and backward priming effects in naming and lexical decision tasks using asymmetrically related words, compound cue words, and purely semantically related words. In the lexical decision and naming tasks, backward and forward priming effects were equivalent for asymmetrically related words and semantically related words.

A major weakness of the study was that compound cues were tested only in a naming task and no priming effects were found in either direction. Despite this

\footnotetext{
${ }^{2}$ Based on association strength values available from Nelson, McEvoy, and Schreiber's (1998) University of South Florida Free Association Norms online database.
} 
inconsistency, Thompson-Schill et al. (1998) concluded that semantic relatedness is both sufficient and necessary for producing forward and backward priming under automatic processing conditions. Their reasoning was based on the fact that compound cue words having no semantic relation produced no priming effects under automatic processing conditions, and the fact that backward association strength is virtually non-existent for most compound cues and asymmetric associates. Moreover, the researchers also concluded that the associative relation between related words has no impact on priming under automatic processing conditions. These conclusions were based on the fact that asymmetrically related words have much stronger association strengths in the forward direction, yet the priming effect was comparable in both priming directions

Hutchinson (2003) has questioned Thompson-Schill et al.'s results because they used their own procedure to determine association strengths for asymmetrically related items used in this study. However, the researchers did not use this procedure to measure association strength values for the compound cues they borrowed from Seidenberg et al.'s (1984) study. Using Nelson et al.'s (1998) association norms, Hutchinson (2003) showed that the association strengths in both directions were higher on average for the asymmetric associates than for the compound-cue words used in the study. As a result, the role of association strength in both forward and backward priming for asymmetrically related words cannot be ruled out completely.

Despite this criticism of Thompson-Schill et al.'s (1998) study, there has been support from Chwilla et al.'s (1998)'s study in Table 1 for the role of semantic relatedness in producing backward priming under automatic processing conditions. In addition to measuring reaction times in the study's lexical decision task, the researchers 
also examined N400 event-related potentials (ERP) in the brain during the task. N400 studies of semantic priming are based on examining the negative going ERP shift that occurs in the brain after the stimulus onset of the target word. Compared to unrelated words, this negative going shift is usually much smaller or absent for purely semantically related items (Bentin, McCarthy, \& Wood, 1985). The effect of associative relatedness compared to semantic relatedness on N400 priming effects is still unclear because of inconsistency in the selection and control for semantic relatedness in associatively related items (Hagoort, Brown, \& Swaab, 1996; Koivisto \& Revonsuo, 2001). Chwilla et al. found N400 priming effects under both forward and backward priming conditions at both short and long SOAs, but the related items used by the researchers consisted of both asymmetric associates and compound cues. However, the strongest N400 priming effects in both directions occurred for purely semantically related items. Despite these findings, the forward and backward priming effects at the short SOA involved a lexical decision task with a high RP and NR. Consequently, it is unclear whether to attribute Chwilla et al.'s forward and backward priming results as the product of automatic or strategic processing mechanisms.

Semantic relatedness may not be the sole variable contributing to backward priming effects for asymmetrically associated words. MacNamara (2005) proposed that the target word's lexical frequency may be an important factor because studies find an interaction between the lexical frequency of a target word and the context of its presentation. Low frequency target words yield larger priming effects than higher frequency target words under automatic processing conditions (Becker, 1979; Stone \& Van Ordern, 1992). The relevance for backward priming studies is that the target words 
tend to be of lower frequency in the backward priming direction than in the forward direction (e.g., CROWN is a lower frequency word than is KING). But, if lexical frequency affects backward priming, then there should be a greater magnitude for backward priming than for forward priming under automatic processing conditions. Based on Thompson-Schill et al.'s (1998) results discussed earlier, it is the semantic relationship between asymmetric associates that produces both forward and backward priming. Forward and backward priming effects are equivalent because the semantic relationship contributes equally in both priming directions. However, if the lexical frequency of the target word is also factored into this account, the magnitude of backward priming should be greater than forward priming because it benefits from the additional contribution of typically lower target word frequencies. This is problematic because it goes against the fact that most priming studies presented in Table 1 show greater priming magnitudes in the forward direction.

The explanation for the fact that backward priming is not stronger than forward priming for asymmetrical associates is that the forward associative relationship for asymmetrical associates is typically much stronger than in the backward priming direction. As mentioned earlier, related word pairs that have an associative and semantic relationship generally produce the strongest priming effects. This combination of a strong forward association strength and a semantic relation is, thus, responsible for producing stronger forward priming than backward priming in asymmetrically associated word pairs.

To summarize, forward priming in asymmetrically associated words is produced by the combined influence of the associative and the semantic relations between a related 
word pair. In backward priming, the semantic relationship and lower lexical frequency of the target word are contributing factors, and the contribution of the associative relationship is much weaker or non-existent. The degree of influence that lexical target word frequency and association strength have on priming is unknown. As a result, this account of the underlying mechanisms involved in forward and backward priming for asymmetrical associates does not resolve the issue of which priming direction will produce the strongest priming effects.

Due to the methodological weaknesses and inconsistencies in previous studies, more research is needed in order to determine the cognitive mechanisms and lexical information factors that contribute towards backward priming in asymmetrically associated word pairs. First, there is a need to identify whether automatic or strategic processing mechanișms contribute to backward priming effects. Second, it is necessary to ascertain how backward priming in an asymmetrically associated word pair is affected by the lexical target word frequency, semantic relatedness, and the word pair's associative relationship. The current study aimed to resolve both of these issues and, thus, provide a more detailed account on how backward priming arises in asymmetrically associated words pairs.

\section{Literature Review of Neural Networks}

Artificial neural network models have become a major area of research in both artificial intelligence and cognitive science since the mid 1980s. Another term used for artificial neural networks is parallel distributed processing. Neural networks perform simultaneous (parallel) operations upon several pieces information and the representation of larger pieces of information is distributed amongst several processing nodes. 
Neural network models are inspired from the biological architecture and processing dynamics used by the neurons in the brain. The human brain itself is essentially composed of a multitude of neurons interconnected with one another, whereby information is transmitted via electrochemical signals from one neuron to another. Taken on a larger scale, groups of neurons sending these signals back and forth to one another and other parts of the brain allow patterns of information to form and interact with one another. This pattern-based neural activity in the brain is assumed to form the basis of our sensory, perceptual, and cognitive processes.

Neural network models are able to learn to represent and process information in a similar manner but are not considered exact replicas of the neural dynamics in the human brain. Although artificial neural networks do share some of the architectural and behavioural characteristics of the networks of real biological neurons, the two differ in many ways. In particular, artificial neural networks are considered much simpler in design and processing dynamics compared to their biological counterparts. For this reason, cognitive scientists who study neural networks usually consider these artificial models replicating the mental functioning that exists between the neurobiological level (the lowest level) and the level of conscious thought and awareness (the highest level). This level of functioning has been termed "sub-symbolic" level (Smolensky, 1995) since conscious thought and reasoning are considered to be symbolic in nature (i.e., similar in form and manipulation as a natural language syntax or symbolic logic).

Another way to understand the role of neural networks in mental processing is the through the "tri-level hypothesis" of mental functioning. Marr (1982) formulated three levels of description for mental functions that are necessary to adequately describe how a 
certain mental process is carried out. The highest level is the computational level. At this level, the purpose is to explain what the goal of the process is and the logic of the mental strategies being implemented. The middle level is called the algorithmic level and its purpose is to describe how this process is executed and the necessary representation of the input and output data required during execution. The physical level is the last level and it provides a description of the physical components (i.e., neurobiological) required to perform the operation. Neural networks are considered to provide a description at the algorithmic level. Neural network models, therefore, may be conceived of as a level of mental representation that bridges the explanation gap needed to define the relation between the human brain's neurobiological level of activity and its highest levels of cognitive functioning.

\section{Neural Network Models of Semantic Priming}

A number of neural network models of semantic priming have been proposed, but these models have for the most part focused on forward direction priming between associatively related words or symmetrical priming that occurs between purely semantically related words (e.g., Becker, Moscovitch, Behrmann, \& Joordens, 1997; Cree, McRae, \& McNorgan, 1999; Masson, 1995). In general, there has been little effort made towards producing a model that displays backward priming effects for associatively related words. One model that did investigate backward priming was a continuous-time recurrent neural network (Plaut, 1995). Plaut's model was capable of demonstrating priming effects in both purely semantically related words and between associatively related words. The neural network also tested for the effects of lexical frequency, the degree of semantic relatedness, and SOA. 
Plaut's (1995) neural network was trained by having words in a pair presented sequentially and then tested for priming effects. The associated word patterns did not have any semantic overlap but, during training, were presented together sequentially in greater frequency than any other possible sequential pairing from the network's word set. However, these associatively related word-pairs appeared in only one sequential direction during the training sequences.

Plaut (1995) found that the model yielded no backward priming effects. In a similar model developed by Plaut and Booth (2000), related words shared both an associative and a semantic relation. However, backward priming was not investigated. This was due to the fact that, during training, the researchers allowed an equal probability that associatively related words were presented in both directions. The researchers speculated that, based on the null results with Plaut's (1995) earlier model, any backward priming effect was a result of a shared semantic overlap held between associatively related words. Therefore, a study that investigates the contribution of a semantic relation towards backward priming is needed.

Moss, Hare, Day, and Tyler (1994) developed a recurrent neural network model that was capable of displaying priming for both associatively related and semantically related words. The processing mechanisms in Moss et al. were simpler than those in Plaut (1995). Moreover, the time factor was represented in a discrete manner rather than in a continuous one. The type of neural network used by Moss et al. (1994) is called a simple recurrent neural network (SRNN) and followed an architectural design based on Elman's (1990) model. Elman's network was designed to incorporate a time processing 
component that would allow the neural network to form sequential relations between word patterns based on their order of presentation.

Elman's SRNN model was adopted by Moss et al. (1994) to determine whether it was capable of demonstrating the effects of semantic priming in a word naming task. More specifically, the researchers assessed whether the model was capable of producing the different magnitudes of priming found in empirical studies of word pairs that were associatively (but not semantically) related, semantically related, or related in both ways. Moss et al.'s testing method for priming effects involved the presentation of word pairs in an immediate sequence (i.e., there was no time lag between the presentation of the prime and target). That is equivalent to using a short SOA in human priming studies. The priming effect for associatively related words was significant, but there was no difference between purely associatively related words and associatively/semantically related words (Moss et al., 1994). Purely semantically related items did produce some priming compared to unrelated words, but the effect was not significant. However, the researchers did not examine the effect that different degrees of semantic overlap had on priming for purely semantically and associatively/semantically related items. Thus, it is premature to draw any conclusions about the role of semantic relatedness in producing the neural network's priming effects.

\section{Current Study}

In order to provide a more conclusive account of backward priming for asymmetrically associated words, the current study conducted a lexical decision task experiment using an set of asymmetrically associated word pairs drawn from those used in previous backward priming studies. The performance of the human participants in this 
experiment was then compared with the performance of an SRNN model in a comparable lexical decision task. Compound cues were not included in the study because the current study modeled backward priming effects in a neural network. Developing and training compound cue pairs in the Moss et al. (1994) model is problematic. Non-semantically related words trained in that model actually had a large degree of semantic overlap between them. ${ }^{3}$ The possibility of training compound cue pairs in an SRNN is addressed in the general discussion.

In addition to forward and backward priming effects, the role of association strength and semantic relatedness in forward and backward priming were also examined. Association strength between a related word pair is measured by recording how often one of these words comes to mind when an individual is presented with the other word. Semantic relatedness refers to the number of semantic features shared by two words. Although association strength values are available via studies of word association norms, there does not exist an objective measure for semantic relatedness. It is possible to determine the effects of semantic relatedness by using Thompson-Schill et al.'s (1998) approach of comparing the magnitudes of forward priming and backward priming for word pairs that have a forward association strength but no backward association strength.

\footnotetext{
${ }^{3}$ In Moss et al.'s (1994) model, a word's semantic representation consisted of vector pattern of 1 's and 0 's. A value of 1 represented an active semantic feature and a value of 0 represented an inactive semantic feature. For every word used in the study, the majority of semantic features were inactive. Semantic relatedness between two words was defined by the number of active semantic features they shared. Semantically related words shared a larger number of active semantic features than non-semantically related words. However, due to the nature of the processing mechanisms in an SRNN, both the active and inactive semantic features shared by any given word pair are in fact treated as semantically related features in this type of neural network model. Moss et al. failed to consider this when they generated the non-semantically related words for their SRNN. Because most semantic features were inactive between any given nonsemantically related word pair, it arguable that there was a high degree of semantic relatedness between the words in most of these word pairs.
} 
If there is no difference in priming magnitudes, then it can be inferred that semantic relatedness is responsible for producing priming effects in both directions. If this approach is used, however, it must address the problems raised by Hutchinson (2003) concerning Thompson-Schill et al.'s measures of backward association strength between related word pairs. Furthermore, Thompson-Schill et al.'s approach cannot provide an answer as to whether or not the degree of semantic relatedness between asymmetrically associated words influences the magnitude of forward and backward priming.

Unlike previous backward priming studies, the current study examined the effect of the degree of semantic relatedness between related word pairs on priming. In general, measures of semantic relatedness between related words have not been used in semantic priming studies because it is difficult to find a proper method to measure semantic relatedness. McCrae, De Sa, and Seidenberg (1997) and Shelton and Martin (1992) measured semantic relatedness between related items by having participants rate their similarity. Lucas (2000) has argued that this approach suffers from issues of reliability and objectivity because there has been no standardization between research studies on the procedures and measurements employed in these judgment tasks. Therefore, a more reliable method would require a more objective means of measuring semantic similarity.

With the need for objectivity and reliability in mind, the current study measured semantic relatedness between related words using lexical semantic information provided by the WordNet lexical database. This database groups word meanings into cognitive synonyms or "synsets" that represent distinct concepts (Miller, Fellbaum, Tengi, Wakefield, Poddar, Langone, \& Haskell, 2006). Synsets are organized within a hierarchy composed of "is-a" hypernym features or super-ordinate sysnets that all words in the 
synset have in common. Different sysnets can be linked to one other by having a common super-ordinate synset within the hierarchy. Based on the original WordNet database, Pedersen, Patwardhan, and Michelizzi (2004) developed a computer program called WordNet::Similarity, designed for measuring semantic similarity between word pairs. The semantic similarity between two words is calculated using a distance metric that analyzes the proximity between each word's sysnet within the hierarchy.

Evidence for an association strength effect on priming in semantic priming studies has been mixed (Anaki \& Henik, 2003). Koriat (1981) found that associative strength did not influence forward and backward priming effects, but association strength was measured by the participant's word association judgments. Therefore, Koriat's results are subject to the validity and reliability of this measure of association strength. The current study used association strength values provided by the more established University of South Florida Free Association Norms online database created by Nelson, et al. (1998). For the current study's statistical analysis of the effects of association strength on priming, a multiple regression analysis was used. Because almost half of the related word pairs used in the current study have a backward association strength value of 0 , the role of association strength in backward priming could not be analyzed statistically (i.e., backward association strength values could not be transformed to form a normal distribution of values). Instead, the role of forward association strength on forward and backward priming was examined. No previous study has considered the possibility that the forward association strength between asymmetrically related word pairs contributes towards backward priming.

Lexical frequency has shown to be a determining characteristic in semantic 
priming studies, with low frequency target words yielding greater priming effects than high frequency targets. The lexical frequency effect of target words in backward priming was examined in the present study. Furthermore, it was possible to integrate lexical frequency into the training of the current study's neural network simulation and determine if the model could produce results comparable to the human participants in the experiment.

The current study's methodology followed the procedure of Thompson-Schill et al. (1998) with modifications. It tested two levels of the SOA and removed the neutral target word condition. The removal of the neutral condition created a non-word presentation ratio of 0.50 , a ratio that is more conducive to eliciting automatic processing in a lexical decision task (MacNamara, 2005). The relatedness proportion (0.50) was the same as in Thompson-Schill et al. Another modification was the addition of asymmetrically associated word pairs drawn from a number of previous backward priming studies including the set used by Thompson-Schill et al. (1998). Predictions for the Experiment

For the experimental component of the study, there are a number of specific predictions about the type of priming effects that will be demonstrated by human participants. The role of strategic and automatic lexical processing mechanisms in backward priming was investigated by varying the SOA. The RP and NR values were kept constant across the SOA condition. Similar to Thompson-Schill et al.'s (1998) study, the short SOA condition in the current experiment was expected to produce equal magnitudes of priming in both priming directions. In the case of the long SOA condition, it was expected that there would be more priming in the forward direction than in the 
backward direction. The reason for this prediction is (as shown in Table 1) that it coincides with the fact that asymmetrical priming effects have mostly been found in previous studies that had experimental conditions favouring strategic processing. It is also predicted that priming in both directions will be greater at a shorter SOA since asymmetrically related words rely more heavily on automatic processing for their priming effects.

As shown in Table 3, target item frequencies tend to be lower in the backward direction (prime words in Table 3 are target words in the backward direction). Given that low frequency target items elicit stronger priming effects (e.g., Becker, 1979; Stone \& Van Orden, 1992), it is hypothesized that the low frequency target words will yield greater forward and backward priming than high frequency target words. Finally, it is predicted that semantic relatedness plays a significant role in producing both forward and backward priming effects. In particular, it is hypothesized that related word pairs with a greater degree of semantic relatedness will produce greater magnitudes of priming in the forward and backward priming directions.

Predictions for the Neural Network Model

The simulation part of the study attempted to provide a neural network model of backward priming using asymmetrically associated words. Plaut's (1995) model has already been shown to successfully model a variety of empirical phenomena related to human semantic priming, but has yet to demonstrate its ability to produce backward priming. If the current SRNN model can produce forward and backward priming effects similar to human performance, then it will become a rival to Plaut's neural network model of human semantic priming. If so, it remains to determine which of the two neural 
network's is a better model of human lexical processing. Both models are considered to have a certain degree of cognitive validity. Due to the ability to represent the temporal and dynamical components involved in human information processing, it has been argued that both types of recurrent neural network models have far greater cognitive and neurobiological plausibility than the more popular types of neural networks (e.g., the multi-layer perceptron model) used by cognitive scientists (van Gelder \& Port, 1995). Another criterion for judging competing neural network models (and scientific models in general) is to accept the more simpler of the two if it can account for all the same findings as the more complex one (O'Reilly \& Munkata, 2003). Accordingly, the SRNN uses a less complex method for representing temporal information within its processing mechanisms than does the recurrent neural network used by Plaut (1995).

Similar to the experiment, the SRNN model was tested for forward and backward priming at short and long SOAs. The effects of the lexical frequency of the target word and semantic relatedness were also examined. The associative relationship between a related word pair was formed during the neural network's training phase. Plaut's (1995) method for developing an associative relation between related words was implemented such that related words appeared in sequence more often than did unrelated word pairs, but in only one direction. The unidirectional co-occurrence of related words produced the stronger association strength found in the forward direction for asymmetrically associated words. The absence of a backward association between related items provided a test of whether or not semantic relatedness alone could produce backward priming in the neural network. The current study's SRNN model adopted a similar approach by using only related words without a backward association strength. As done in both 
Plaut's and Moss et al.'s studies (1994), forward association strength was kept constant for all related words. The reasons for not varying the degree of association strength in both priming directions is discussed in further detail the simulation section of the paper. However, the neural network's related word pairs could still be compared to a subset of the human experiment's asymmetrically associated words that had comparable association strength values.

The predictions for the neural network's performance were that it would display backward priming effects at both SOAs, but that it would weaker than forward priming effects. Moss et al. (1994) have already shown that the associative relation between words is mainly responsible for producing priming. As stated earlier, Plaut and Booth (1996) conjectured that semantic overlap between related words is necessary for producing backward priming effects in their neural network. MacNamara (2005) also claimed that the semantic relation shared between asymmetrically associated words is partially responsible for producing forward and backward priming, particularly, at shorter SOAs. Thus, the prediction in the current study is that for related words at the short SOA, the associative relation produces forward priming and the semantic relation produces backward priming. This prediction is similar at the long SOA too, but the influence of the semantic and associative relation between related words will be much weaker and, therefore, priming in both directions will be weaker at the long SOA than in the short SOA. 
The Experiment

\section{Method}

\section{Participants}

The participants were 82 undergraduate students who received a credit towards the research component of their introductory psychology course. One participant was removed from the analysis because of a high rate of incorrect responses (42\%) during the experiment. All of the remaining participants had error rates below 20\%. Participants were assigned randomly to either the short SOA $(n=40)$ or long SOA $(n=41)$ experimental condition.

\section{Stimuli}

Word pairs were selected from items used in prior studies of backward priming (Kahan et al., 1999; Peterson \& Simpson, 1989; Thompson-Schill et al., 1998). There were 55 semantically/asymmetrically associated word pairs with association strengths provided by Nelson et al.'s (1998) University of South Florida Free Association Norms online database and lexical frequency values provided by Kucera and Francis's (1967) word norms. Semantic relatedness between word pairs was measured using Pedersen et al.'s (2004) WordNet::Similarity software program. The 'gloss vector' distance metric was chosen from the available distance metrics because it has been shown to have the strongest correlations with human similarity judgments (Patwardhan \& Pedersen, 2006). Table 3 provides the list of asymmetrically related word pairs, their association strengths, semantic relatedness, and word frequencies.

As in Kahan et al. (1999), the forward priming direction for a related word pair always had a greater association strength than the backward priming direction. Overall, 
association strength was greater in the forward priming direction $(M=0.29, S D=0.20)$ than in the backward priming direction $(M=0.07, S D=0.14)$. The difference between these mean association strength values was statistically significant, $t(54)=9.22, p<.001$. The lexical frequency of the target words was higher in the forward priming direction ( $M$ $=129.95, S D=156.27)$ than in the backward priming direction $(M=35.91, S D=53.91)$. The difference between these mean target word frequency values was statistically significant, $t(54)=4.77, p<.001$. The average semantic relatedness between related word pairs was $0.36(S D=0.19)$.

The effects of association strength, semantic relatedness, and lexical target word frequency on priming were analyzed using a multiple regression analysis. Thus, it was necessary to ensure that these values were normally distributed. The KolmogorovSmirnov ( $k s)$ test for normality showed that the lexical target word frequencies were not normally distributed in the forward $(k s=0.25, p<.001)$ or backward $(k s=0.26, p<$ $.001)$ priming directions. Semantic relatedness $(k s=0.09, p=.20)$ and forward association strength $(k s=0.09, p=.20)$ were both normally distributed. Therefore, lexical target word frequency applied the logarithmic transformation (frequency $=40+$ $\left.\left(\log _{10}(f+1)\right)\right)$ used by Plaut and Booth (2000). This resulted in a mean target frequency of $42.82(S D=0.57)$ in the forward direction and a mean target frequency of $42.25(S D=$ $0.57)$ in the backward direction. The difference between these mean transformed target word frequency values was statistically significant, $t(54)=6.87, p<.001$. Tests for normality indicated that the transformed lexical frequency values were normally distributed in the forward $(k s=0.11, p=.17)$ and backward $(k s=0.09, p=.20)$ priming directions. 
In addition to the related word pairs, 110 unrelated prime words and 110 target non-words were used. Each unrelated prime word was assigned to a related word pair and was matched to have the same number of letters as the related word's prime word. Non-words were generated by changing one to three consonants in each of the existing word in the related word pair set. Another set of 12 unrelated word pairs and 12 word/non-word pairs was created in order to serve as practice trials.

\section{Materials}

Each related word pair formed a base for eight possible test pair conditions. For each base set, two non-words and unrelated words were generated. An example of one of these sets is shown in Table 4. The sets were assigned to testing blocks such that each block consisted of 55 trials. Within a testing block, participants were presented one of the word pair conditions (randomly selected) from each set (one trial). When selecting a trial from a particular word pair set, selection was done exclusively from either the word pair's forward priming conditions or backward priming conditions for each participant. In other words, each participant was subjected to either only forward priming trials or backward priming trials for a given base word pair. The entire experiment consisted of four test blocks comprising 220 trials overall.

\section{Procedure}

The experiment was run using the E-Prime software program (Psychology Software Tools Inc., 2000). Participants were assigned randomly to either the short SOA $(150 \mathrm{~ms})$ condition or the long SOA $(500 \mathrm{~ms})$ condition. The participant was seated approximately $60 \mathrm{~cm}$ in front of an NEC MultiSync LCD 19 inch computer monitor and instructions were presented on the screen. The participant engaged in a practice block of 
24 trials consisting of 12 unrelated word and 12 non-word trials. Following the practice trials, the participant completed four test blocks. Between each test block, the participant was allowed to take a break and pressed a key when they were ready to begin the next test block.

Each trial began with a fixation point (cross) centred on the screen for $500 \mathrm{~ms}$. Next, the prime word appeared 0.80 centimeters above the centre of screen while the target item appeared in the centre. In the short SOA condition $(150 \mathrm{~ms})$, the prime appeared for $150 \mathrm{~ms}$ immediately followed by the target item. In the long SOA condition (500 ms), the prime word appeared for $150 \mathrm{~ms}$ followed by a blank screen for $350 \mathrm{~ms}$. Finally, the target word appeared. The participant was given a time limit of $2000 \mathrm{~ms}$ to decide whether or not the target item was a word or non-word. The participant indicated a decision by pressing the " " key for a word or the "z" key for a non-word on the keyboard. When the participant responded or $2000 \mathrm{~ms}$ elapsed, the next trial began. After the completion of a test block, the participant was allowed to take a short break before proceeding with the next test block. The experiment ended when the participant had completed the four test blocks.

\section{Experimental Results}

As in all previous backward priming studies using asymmetrically related words (Kahan et al., 1999; Koriat, 1981; Peterson \& Simpson, 1989; Thompson-Schill et al., 1998), the current data analysis examined decision latencies for correct "word" responses only. Mean correct latencies for target word responses were calculated for each participant and for each word pair. The main analysis consisted of a $2 \times 2 \times 2 \times 2$ (SOA $\times$ Test Blocks $\mathrm{x}$ Priming Direction $\mathrm{x}$ Prime Type) ANOVA. For the Test Blocks factor, the 
latencies from the first two and last two test blocks were compared. This was due to the fact that, for each participant, the experiment had multiple presentations of the same prime and target words. Therefore, it was necessary to test whether this item repetition affected decision latencies over test blocks. Table 5 presents the mean reaction times for all target word conditions in the experiment collapsed across participants and word items.

The data analysis also examined the percentage of response errors made by participants for target word trials. Table 6 presents the percentage of response errors for all target word conditions in the experiment collapsed across participants and word items. The error response analysis consisted of a $2 \times 2 \times 2$ (SOA $\times$ Priming Direction $\times$ Prime Type) ANOVA. The Test Blocks factor was omitted due to the fact that the number of presented target word trials and correct responses to these varied considerably across participants and test blocks.

For both the decision latency and response error ANOVAs, participants $(F 1)$ and word items $(F 2)$ were treated as random factors. SOA was treated as a between-subjects effect in $F 1$ and as a within-items effect in $F 2$. All of the remaining factors were treated as within-subjects and within-items effects. Unless otherwise stated in the results section, only significant results are reported.

Table 7 and Table 8 provide the ANOVA results for decision latencies across subjects and word items. The analysis of variance for decision latencies revealed that there was a significant main effect for SOA across participants, $F 1(1,79)=7.18, p<.01$, and across word items, $F 2(1,54)=221.26, p<.001$, indicating that the long SOA latencies $(M=603)$ were shorter than the short SOA latencies $(M=658)$. A significant main effect for testing blocks was found at both the participant, $F 1(1,79)=4.42, p<.05$, 
and word item levels, $F 2(1,54)=9.70, p<.01$. This significant main effect showed that decision latencies were shorter in the last two test blocks $(M=625)$ than in the first two $(M=636)$. The priming direction was also significant for both participants, $F 1(1,79)=$ $52.50, p<.001$, and word items, $F 2(1,54)=21.96, p<.001$, with shorter latencies in the forward direction $(M=614)$ than in the backward direction $(M=647)$. Most importantly, related word pairs $(M=624)$ yielded shorter decision latencies than did unrelated pairs $(M=637)$ for participants, $F 1(1,79)=17.23, p<.001$, and for word items, $F 2(1,54)=$ $21.57, p<.001$. A significant interaction effect was found for SOA $\times$ Block $\times$ Direction for participants, $F 1(1,79)=6.18, p<.05$, but was marginally significant for word items, $F 2(1,54)=3.70, p=.06$. No other interaction effects were statistically significant.

Although the SOA $x$ Block $x$ Direction interaction was marginally significant for word items, its simple effects were examined. In the first two test blocks at the short SOA, forward priming latencies $(M=643)$ were shorter than backward priming latencies $(M=689)$ for both the participant, $F 1(1,79)=34.92, p<.001$, and word item levels, $F 2(1,109)=23.79, p<.001$. The last two test blocks at the short SOA showed that forward priming latencies $(M=638)$ were shorter than backward priming latencies $(M=$ 662 ) for both the participant, $F 1(1,79)=12.06, p<.01$, and word item levels, $F 2(1,109)$ $=11.03, p<.01$. In the first two test blocks at the long SOA, forward priming latencies $(M=593)$ were shorter than backward priming latencies $(M=618)$ for both the participant, $F 1(1,81)=9.90, p<.01$, and word item levels, $F 2(1,109)=9.45, p<.01$. The last two test blocks at the long SOA showed that forward priming latencies $(M=$ $581)$ were shorter than backward priming latencies $(M=618)$ for both the participant, $F 1(1,81)=22.82, p<.001$, and word item levels, $F 2(1,109)=20.02, p<.001$. 
Table 9 and Table 10 provide the ANOVA results for error rates across subjects and word items. The analysis of variance for the percentage of response errors did not show a significant main effect for SOA for participants, $F 1(1,79)=1.18, p=.28$, or for word items, $F 2(1,54)=1.42, p=.24$. For both participants, $F 1(1,79)=8.42, p<.01$, and word items, $F 2(1,54)=7.20, p<.05$, there was a significant main effect for priming direction. There was a lower error rate in the forward priming direction $(M=2.59)$ than in the backward priming direction $(M=4.18)$. The main effect for Prime Type was statistically significant for participants, $F 1(1,79)=21.98, p<.001$, and word items, $F 2(1,54)=13.32, p<.01$. Related word pairs had lower error rates $(M=2.61)$ than did unrelated word pairs $(M=4.15)$. No interaction effects were statistically significant.

Part of Thompson-Schill et al.'s (1998) data analysis examined decision latencies from only the first test block. This enabled the researchers to analyze decision latencies from each participant prior to any potential influence from target item repetition. Thus, the present study conducted a $2 \times 2 \times 2$ (SOA $\times$ Priming Direction $\times$ Prime Type) ANOVA using decision latencies from only the first test block. For the word item analysis, four items were removed due to insufficient data in the first test block $(N=51)$. Significant effects were found for SOA for participants, $F 1(1,79)=5.68, p<.05$, and items, $F 2(1,50)=45.78, p<.001$. There were shorter latencies at the long SOA $(M=$ $602)$ than at the short SOA $(M=658)$. Priming direction was also significant for participants, $F 1(1,79)=10.51, p<.01$, and items, $F 2(1,50)=4.97, p<.05$. Forward priming decision latencies were shorter $(M=617)$ than backward priming latencies $(M=$ 642). Prime relatedness was not significant for participants, $F 1(1,79)=3.22, p=.08$, or word items, $F 2(1,50)=3.29, p=.08$, but latencies were still comparable to those found 
in the ANOVA using all test blocks Latencies for related word pairs $(M=623)$ were shorter than unrelated word pair $(M=637)$ latencies. No interaction effects were statistically significant.

Many of the related word pairs had a backward association strength value greater than $0(n=29)$ and some words had a very weak $(<.05)$ forward association strength $(n=$ 5). Therefore, it is difficult to determine if the backward association strength or very weak forward association strength actually contributed towards backward priming. To rule out the effects of backward association strength on backward priming, a second ANOVA was run using only related words that had a backward association strength of 0 and a minimum forward association strength of $0.05(N=21)$. One participant was removed from the participant-based $(F 1)$ ANOVA due to insufficient data. Overall, the effects in the analysis using this subset of related words were similar to those in the in the analysis using the entire word set. There was a significant main effect for SOA for participants, $F 1(1,78)=7.97, p<.01$, and for word items, $F 2(1,20)=110.42, p<.001$. Latencies were shorter at the long SOA $(M=607)$ than the short SOA $(M=671)$. The main effect for testing blocks was not significant for participants, $F 1(1,78)=3.61, p=$ .06 , but was significant for items, $F 2(1,20)=4.53, p<.05$. A statistically significant main effect for priming direction was found for both participants, $F 1(1,78)=73.16, p<$ .001 , and for word items, $F 2(1,17)=17.66, p<.001$. The forward priming direction $(M$ $=608)$ yielded shorter latencies than did the backward direction $(M=670)$. There was a significant main effect for prime type for participants, $F 1(1,78)=6.01, p<.05$, and for word items, $F 2(1,20)=6.57, p<.05$. Related word paịs $(M=629)$ had shorter latencies than unrelated word pairs $(M=648)$. No interaction effects were statistically significant. 
Association Strength, Semantic Relatedness, and Lexical Frequency

The final data analysis examined the roles of target word frequency, forward association strength, and semantic relatedness on priming effects. A series of multiple regression analyses was conducted in order to determine the relationship between these variables and forward and backward priming. The analysis used the forward association strength values, semantic relatedness values, and the logarithmically transformed lexical frequency values of the target words as the predictor variables. For the outcome variable, the magnitude of priming for each related word pair (i.e., the difference between the average latencies of the unrelated word pair condition and the related word pair condition) was used. The bivariate and partial correlations of the two predictors with forward priming at both SOAs are presented in Table 11. The strongest correlation was found between forward association strength and forward priming at the short SOA, but it was not statistically significant $(r=0.17, p=.22)$. None of the other predictor variables were significantly correlated with forward priming at both SOAs. Table 12 provides the summary of the regression analyses for forward priming at both SOAs. Forward association strength at the accounted for the largest proportion of variance amongst all of the predictor variables for forward priming, but it was not statistically significant $\left(R^{2}=\right.$ $0.03), F(1,53)=1.52, p=.22$. Overall, the regression model showed that none of the predictor variables could account for a statistically significant proportion of the variance in forward priming. The backward priming bivariate and partial correlations are presented in Table 13. Table 14 summarizes the regression models for backward priming at both SOAs. At the long SOA, target word frequency was both significantly correlated $(r=-0.27, p<.05)$ and accounted for a statistically significant proportion of the variance 
$\left(R^{2}=0.07\right), F(1,53)=4.11, p<.05$. The lower the frequency of target words, the greater the magnitude of backward priming.

A second series of multiple regression analyses was run using the subset of related word pairs that had no backward association strength and a minimum forward association strength of 0.05 . This was done in order to ensure the complete separation of the influences that forward and backward association strength might have on backward priming. Table 15 presents the bivariate and partial correlations of the predictor variables with forward priming at both SOAs using this subset of related word pairs. The strongest correlation was found between semantic relatedness and forward priming at the long SOA, but it was not statistically significant $(r=-0.24, p=0.31)$. None of the other predictor variables were significantly correlated with forward priming at both SOAs for the subset of related words. Table 16 provides the summary of the regression analyses for forward priming at both SOAs. Semantic relatedness at the long SOA accounted for the largest proportion of variance amongst all of the predictor variables for forward priming, but it was not statistically significant, $R^{2}=0.06, F(1,53)=1.11, p=.31$. Overall, the regression model showed that none of the predictor variables could account for a statistically significant proportion of the variance in forward priming using the subset of related words. For backward priming in this subset of related word pairs, the bivariate and partial correlations with the predictor variables at both SOAs are presented in Table 17. Both target word frequency $(r=-0.50, p<.05)$ and forward association strength $(r=0.53, p<.05)$ were significantly correlated with the magnitude of backward priming. Lower target word frequencies and higher forward association strength produced greater magnitudes of backward priming. Table 18 summarizes the regression 
models for backward priming at both SOAs. As shown in Table 18, at the short SOA, all three variables together accounted for a statistically significant amount of variance $\left(R^{2}=\right.$ $0.38), F(3,17)=3.52, p<.05$, for backward priming effects. Furthermore, lexical target word frequency accounted for a statistically significant amount of variance $\left(R^{2}=0.25\right)$, $F(1,19)=6.22, p<.05$, and so did forward association strength variance $\left(R^{2}=0.28\right)$, $F(1,19)=7.37, p<.05$, for backward priming effects.

\section{Discussion}

The forward and backward priming effects were equivalent in magnitude replicating the findings by Thompson-Schill et al. (1998). The priming effects did not vary across short and long SOAs replicating the findings by Kahan et al. (1999). These patterns emerged across all items and for the subset of related word pairs that had a minimum forward association strength of 0.05 and no backward association strength. For the subset of related word pairs, stronger forward association strengths led to greater backward priming at the short SOA. Therefore, the associative mechanism involved in backward priming contributes to automatic processing, contrary to Thompson-Schill et al.'s conclusion. A backward associative relation between asymmetrically related words is not required for backward priming effects.

Backward priming effects are elicited by either forward or backward associative mechanisms. If there is a backward association between related items in the backward priming direction, then the word pair will rely upon this association to produce backward priming effects. In the absence of a backward association, the word pair's forward associative relation can produce backward priming effects. This explanation is supported by Seidenberg et al.'s (1984) proposal that, due to the weak or non-existent backward 
association between compound cues, backward priming relies upon a post-lexical relatedness-checking strategy. This post-lexical mechanism searches for an associative relationship between the prime and target in both directions to elicit backward priming effects. In contrast to Seidenberg et al's notion that this post-lexical mechanism is strategic, there is greater evidence to support the view that it operates during automatic processing (Chwilla et al. 1998; De Groot, 1984; De Groot, 1985; Peterson \& Simpson, 1989). Chwilla et al. also demonstrated that asymmetrically associated words are subject to this post-lexical search mechanism in backward priming.

Priming effects occurred at both short and long SOAs, a finding that suggests automatic processing mechanisms underlie priming for asymmetrically associated words. However, the results showed that decision latencies decreased across testing blocks and that priming effects in the first test block were not significant. Both of these results point towards target item-repetition effects. Thompson-Schill et al. (1998) also found that lexical decision latencies decreased across test blocks but their priming effects were present in the first block alone. The non-significant priming results from the current study's first test block may be the result of the lower number and larger variability of available reaction times from each participant. Levene's test for the equality of variances supports this notion. The variance for the mean latencies in the first test block for participants $(S D=123.56)$ was significantly higher than in all test blocks $(S D=108.11)$, $F 1=5.40, p<.05$. This was also the case for word items in the first test block ( $S D=$ 109.48) compared to all test blocks $(S D=76.40), F 2=58.34, p<.001$. Nevertheless, it should be noted that the level of priming in the first test block was numerically similar to that in the overall analysis. 
It may also be argued that target item repetition affected both related and unrelated word pairs equally. This was supported by the fact that no significant interaction was found between the Test Blocks and Prime Type factors. In accordance with this, den Heyer, Goring \& Dannenbring's (1985) priming study found that target item repetition effects are independent of word pair relatedness. Therefore, it is unlikely that the current study's priming effects resulted from item repetition.

Lexical frequency effects were found using the entire set of related word pairs, but these effects were present only in the backward priming direction at the long SOA. Lower frequency target words yielded a greater magnitude of backward priming effects at the long SOA. The lack of an effect of target word frequency on backward priming at the short SOA is consistent with the findings by Stone and Van Orden (1992) that word frequency does not exhibit its effects under automatic priming conditions (shorter SOAs). The item repetition effects of the multiple presentations of the target word in the current study might come into play here because it has been found that the influence of target word frequency on decision latencies decreases across target word repetition (Forbach, Stanners, \& Hochhaus, 1974). However, Forbach et al. also found that target word frequency does not interact with the relatedness between word pairs. Therefore, lexical frequency repetition effects from target words would affect both related and unrelated word pairs equally across test blocks in the current study.

Semantic relatedness did not appear to have any effect on the magnitude of priming in either priming direction. This finding appears contrary to the notion that semantic relatedness contributes toward both forward and backward priming for asymmetrically associated words (Thompson-Schill et al., 1998). In fact, the present 
study indicates the degree of semantic relatedness between asymmetrically associated words has no influence on forward or backward priming. It does not discount the possibility that simply the presence of a semantic relation between a pair of contributes towards priming effects. A second possibility is that the semantic relation between a word pair contributes towards forward or backward priming via an interaction with the associative relationship. In order to evaluate these two possibilities, a future study comparing compound cue and asymmetrically associated word pairs is required. Finally, there is the issue of the semantic relatedness measure's validity. It is possible that the semantic relatedness values provided by WordNet::Similarity do not accurately reflect the cognitive mechanisms that represent the semantic relationship between word pairs. Moreover, unlike the measures for lexical frequency and association strength, there is a larger number of competing measures of semantic relatedness available and there is no general consensus of which to use. Therefore, no definite conclusions can be made from the present study's results regarding the role of semantic relatedness in backward priming for asymmetrically associated words.

The analysis of the subset of related word pairs without a backward association strength and a minimum forward association strength of 0.05 yielded different effects of target word frequency and forward association on backward priming. In this case, both of these variables were significant predictors of the magnitude of backward priming. Lower frequency target words and higher forward association strengths produce greater magnitudes of backward priming at the short SOA only. This frequency effect is inconsistent with findings by Stone and van Orden (1992), who found that lower lexical frequency target words produce greater magnitudes of priming under strategic priming 
conditions. However, it is questionable whether these results can be generalized to a backward priming study because Stone and van Orden examined lexical frequency effects using a forward priming task. The present study's finding provides evidence that lexical frequency effects on backward priming can occur under automatic processing conditions for asymmetrically associated word pairs.

One of the goals of the experiment was to determine the involvement of automatic and strategic processing mechanisms in producing backward priming effects for asymmetrically related words. Besides the presence of backward priming effects at both SOAs, there are other indicators from the current study that support the involvement of automatic processing in backward priming for asymmetric associates. First of all, as Table 5 shows, there was a larger magnitude of backward priming at the short SOA than in the long SOA. Furthermore, the use of a non-word ratio of 0.50 in the experiment eliminated semantic matching and strategic guessing effects. The experiment's relatedness proportion $(0.50)$ is lower than in most previous backward priming studies using asymmetrical associates and, thus, reduces expectancy effects present during strategic processing. Still, an expectancy effects could still be present because an RP of 0.20 or lower has been recommended by MacNamara (2005) in order to thoroughly eliminate expectancy effects in a semantic priming task. It is reasonable to conclude that the majority of evidence points towards automatic processing being primarily involved in producing backward priming effects in asymmetrically related word pairs.

To summarize the research findings of the experiment, it is evident that forward and backward priming effects rely upon automatic processing mechanisms. In backward priming, lower frequency target words contribute towards priming. There is some 
evidence that the associative relation between asymmetrically related words is involved in backward priming, but the role of semantic relatedness has not been determined. Nevertheless, the current experiment's findings have advanced our knowledge of the nature of backward priming in asymmetrically related word pairs.

\section{The Neural Network Model}

A simple recurrent neural network was chosen as the connectionist model to test for backward priming effects. This model is sensitive to the frequency and order of presentation when forming association links between word representations. In Moss et al.'s (1994) model, forward priming was produced by increasing the frequency of presenting related words together in a sequence. Therefore, one option for the present study was to produce forward and backward priming effects in the same manner. Words forming a related word pair would be presented sequentially more frequently in both directions than would words in an unrelated word pair. However, this was not attempted in the current study because it is an artificial way to accomplish a backward association. The validity of a connectionist model's ability to represent a particular cognitive function lies within the nature of its design and processing dynamics, not in the researcher's ability to manipulate a training environment favourable to accomplishing the goals of each task with which it is presented. Hence, as in Plaut (1995), the neural network in the present study was trained with related word pairs presented sequentially in a single direction. If backward priming were to occur, it would be the product of the semantic relation shared between the related word pairs rather than a product of their associative relation. In addition to the effects of semantic relatedness, the contribution that the target word frequency has towards forward and backward priming was also examined in the 
model in order to see if it could produce results similar to those found in the experiment.

Method

\section{Neural Network Architecture}

Moss et al.'s (1994) SRNN model comprised a phonological input layer (66 units), a hidden layer (40 units), a context layer (40 units), and a semantic output layer (59 units). For the purposes of simulating a lexical decision task in the current study, an orthographic input layer replaced the phonological layer and was reduced to the same size as the orthographic layer used in Plaut and Booth's (2000) model. Other than the reduction in the input layer size, the substitution of a phonological input layer (word naming) with an orthographic input layer (lexical decision) has no effect on how the neural network processes input layer information. It was also decided that a larger semantic layer (also the same size as Plaut and Booth's semantic layer) was needed in order to allow greater variability and distinctiveness defining the semantic representations of different word patterns. Subsequently, the hidden and context layers were increased in size, in order to compensate for the expanded semantic layer. The issue of how these adjustments impact on the ability to assess Moss et al.'s original model for backward priming is not pertinent. An assessment should be focused on the ability of an SRNN's processing dynamics and architectural layout (which do not vary between specific instances of an SRNN) to produce backward priming effects. Layer sizes are a matter of preference and requirements of a particular research study.

As shown in Figure 1, the present study's SRNN consisted of an orthographic input layer (30 units), a hidden layer (65 units), a context layer (65 units), and a semantic output layer (100 units). The hidden layer had one-to-one sending weight to the context 
layer connections (each unit in the hidden layer is connected to only one other unit in the context layer) while all other connecting weights in the neural network are fully connected (a unit's sending weight connections are projected to every unit in the receiving layer).

\section{Stimuli}

Overall, a set of 80 word patterns was generated consisting of their orthographic and semantic patterns. The word patterns' orthographic and semantic representations were generated using the Matlab 7.0 programming software (The Mathworks Inc., 2004). Of these 80 words, 24 acted as prime words (in the forward priming direction) with 24 corresponding related target words. The remaining 32 words acted as "filler" words that had no relation to any other word in the entire word set.

The orthographic representation of a word was created by allowing each orthographic unit to have a 0.15 probability of being active (i.e., $=1$ ). Inactive units were set to zero. Each word's orthographic representation had to differ by at least three units from the orthographic representation of any other word. On average, there were 5.88 (SD $=2.12$ ) active orthographic units for the word patterns.

For the semantic representations of prime and filler words, a 100-unit vector representation was generated with a 0.15 probability of a particular unit being active. With the exception of a related prime and target word, each word's semantic representation differed by at least 13 units from the semantic representation of any other word. Each word's semantic vector pattern had an average of $15.01(S D=2.95)$ active units. The semantic representation of each target word was initially assigned the same vector representation of their corresponding prime word. Next, each target word's 
semantic pattern was modified by varying a subset of active and inactive units. Because the degree of semantic overlap for asymmetrically associated words is unknown, half of the related word pairs used in the neural network simulation was designated as high semantic relatedness and the remaining half as low semantic relatedness pairs. Plaut (1995) and Plaut and Booth (2000) used a similar method to generate semantic representations for related words and classified them in a similar manner.

The present study's method for generating the semantic vector representations for high and low relatedness pairs was a slightly modified version of this. ${ }^{4}$ For high semantic overlap word pairs, each active feature for the target word had a 0.2 probability of being set to inactive and a 0.025 probability that an inactive feature become active. A low semantic overlap target word had a 0.4 probability for active features and a 0.05 probability for inactive features. For high semantic overlap word pairs, the prime and target words differed on average by 6.08 features $(S D=2.35)$; low semantic overlap pairs differed on average by 10.33 features $(S D=3.58)$. The difference between these mean semantic overlap values was statistically significant, $t(22)=3.44, p<.01$.

All word patterns were assigned randomly as either high or low frequency words. Half of each of the target, filler, and prime words were designated as high frequency and the remainder as low frequency. In the case of related word pairs, each possible primetarget frequency combination (high-high, high-low, low-high, low-low) was assigned an equal number of high and low semantic relatedness pairs (three of each).

\footnotetext{
${ }^{4}$ In Plaut (1995) and in Plaut and Booth (2000), a related prime and target word were generated from the same "exemplar" semantic vector pattern. Semantic features in high dominance (semantic relatedness) words had a 0.18 probability of being set to inactive and a 0.02 probability for an inactive feature to become active. Low dominance words had a 0.36 probability for active features and a 0.04 probability for inactive features.
} 


\section{Training Procedure}

The neural network simulation was run on a computer using the Mac OSX version 3.2 of the PDP++ neural network software program (O'Reilly, Dawson, \& McClelland, 2003). The neural network's training simulation consisted of the presentation of a series of trials involving two-word sequences. Thirty separate training simulations were run, each consisting of 80,000 trials. The first word presented in a sequence was selected randomly from the entire word list. The probability of selecting high frequency words ( $p \cong 0.0166$ ) was twice that for low frequency words $(p \cong 0.0083$ ). As in Moss et al. (1994), whenever a prime word was selected, there was a 0.25 probability that the second word selected for the sequence would be its related target word. Otherwise, the second word was again selected from the entire word set according to its designated word frequency. If a target word (in the forward priming direction) was selected as the first word, the second word was also selected in this random manner with the exception that the second word selected could not be its related prime word (in the forward priming direction). Thus, a related word pair could only be presented sequentially in one direction during training (i.e., backward association strength $=0$ ). For filler words selected as the first word, the second word was selected randomly taking into account word frequency. In all word pair sequences, the same word could never appear as both the first and second word.

At the beginning of each training simulation, the one-to-one weight connections between the hidden and context layer were set to 1.0 and all the remaining weight connections were initialized uniformly to random values between 0.25 and -0.25 . At the start of each word-pair sequence, the context layer had its units activated to 0.50 , while 
all other layers had their units activated to 0 . Then, the first word's orthographic input was presented and activation values were calculated at each successive layer in the neural network. The hidden layer's activation state was determined by the input it received from the orthographic layer and from the context layer. In turn, the hidden layer sent its own input to the context and semantic layers. The activation value for a receiving unit in any of the network's layers was calculated by taking the sum of the product of each sending unit's activation and its weight connection to the receiving unit. This sum (x) was then transformed into the activation value $y$ that ranged between 0 and 1 (except for the associative and feedback layers) based on the following nonlinear sigmoid function:

$$
y=1 /\left(1+\exp ^{-x}\right)
$$

Since the sending weights from the hidden layer were always set to 1 and were on a one-to-one ratio, the context layer essentially kept a copy of the hidden layer state from the previous time step in the sequence. This was done in Moss et al.'s (1994) neural network. The current neural network, however, had a context layer that retained information from both its previous state and the new input it received from the hidden layer. This type of context layer uses a "hysteresis" factor into the update process of the context's layer activation state. Jordan (1986) introduced this method for updating the context layer's activation state in an SRNN in order to allow the neural network to retain contextual information between events spaced over a longer period of time, such as in the case of a longer SOA. The hysteresis factor in updating the activation state of the contextual layer consisted of combining a proportion of the sending hidden unit value and a proportion of the context unit's current activation in producing its new value. In formal terms, each context unit at time $t$ is updated by the following equation: 


$$
\mathrm{c}_{\mathrm{j}}(t)=h y s t * \mathrm{c}_{\mathrm{j}}(t-l)+(1-h y s t) * \mathrm{~h}_{\mathrm{j}}(t-l)
$$

where $h_{j}$ is the unit from the hidden layer. The hyst value was set to 0.30 in order to allow the new activation state in the context layer to be primarily influenced by the new input being received from the hidden layer, yet still allow some retention of the context layer's previous activation state as well.

When the second word was presented in the sequence, the context layer fed these values back to the hidden layer via its weight connections. This additional input acted as contextual information during the presentation of the second word during the sequence. If a certain ordered pairing of words occurs more frequently than others, then similar contextual information will appear more frequently as well. In turn, this contextual information is factored in more frequently during the calculation of error and weight adjustments, resulting in the neural network being more proficient at "recognizing" a certain target word over others if its related prime word precedes it.

After the semantic layer produced an activation pattern, it was compared to its target or desired semantic activation for the word. The disparity produced an error value for each unit in the semantic layer calculated using the sum of squared errors (SSE) equation averaged across the two words presented in a sequence as shown in equation 3:

$$
S S E=\Sigma_{n}\left(t_{\mathrm{i}}-o_{\mathrm{i}}\right)^{2}
$$

where $t_{\mathrm{i}}$ is the target output for node $i, o_{\mathrm{i}}$ is the actual output at node $i$, and $n$ is the number of units in the output layer. Using these error values, weight connections were updated based on the contribution the units in all previous layers made towards the error for a given output unit averaged across the two word presentations of the sequence. However, the one-to-one weight connections between the hidden and context layers 
always remained at the value of 1 throughout the entire training phase. Weight adjustments were accomplished using the following weight update equation:

$$
\Delta w_{j i}=\eta \delta_{j} x_{j i}
$$

where $\Delta w$ is the change in the weight connecting unit $i$ to $j, \eta$ is the learning rate parameter, $\delta$ is the error associated with unit $j$, and $x$ is the input going from unit $j$ to unit $i$. In the current neural network model, the learning rate was set to 0.03 . The proper term for this method of adjusting weight connections based on error-contribution is called backpropagation and it is the most common form of learning used in neural network.

\section{Testing Procedure}

After each training simulation, weight connection values were frozen and the neural network was presented both related and unrelated word pair conditions. The performance results of the neural network were measured by calculating the sum of the square root of the mean-squared error (root MSE) of the second word's output values during the presentation of a word-pair sequence: The root MSE calculation simulates human response latencies where higher error values represent longer latencies.

Comparing the root MSE calculations between the related and unrelated word-pairs reflects priming. There were 24 tests of each of the each of the four word conditions. There were no non-word conditions though. The forward related condition presented the related prime and target word in the same direction they had appeared during training. The backward related condition presented the same word pairs in reverse order. For the unrelated conditions, 24 filler words (12 high frequency and 12 low frequency) were selected randomly to serve as prime words. Both the forward and backward unrelated word pair conditions were created by replacing the prime word in the corresponding 
related word pair condition with one of these filler words (matching for word frequency of the original). Overall, 30 test phases (one for each of the 30 training simulations) were conducted, and each test phase used the same set of filler words and paired them with the same target words for its unrelated word conditions.

The testing phase also measured the effects of stimulus onset asynchrony using the same word items in both SOA conditions. In the short SOA condition, each primetarget pair was presented in immediate sequential order. In the long SOA condition, the previously used method of having an intervening blank input (no orthographic input) between the presentation of the prime word and target word was implemented (Plaut, 1995; Plaut \& Booth, 2000). Other than having no orthographic input, this phase of the long SOA word pair sequence had the same processing duration as the sequence's prime and target word processing phases. For both the short and long SOA testing conditions, error was calculated after the presentation of the target word.

Neural Network Results

As in Moss et al. (1994), the analysis of the neural network's performance used the square-root of the mean-squared error during testing phase. The average error scores across the different test conditions are reported in Table 19. Error scores were subjected to a $2 \times 2 \times 2$ (SOA $\times$ Priming Direction $\times$ Prime Type) ANOVA. For the analysis, the test results from each training simulation were treated as data from individual participants $(N=30)$. The test results for word items $(N=24)$ were analyzed collapsing across simulations. All of the factors in the ANOVA were treated as within-subject $(F 1)$ and within-item effects $(F 2)$ and both effects were treated as random factors. 
Table 20 and Table 21 summarize the ANOVA results across participants and word items. The main effect of SOA was not significant for participants, $F 1(1,29)=$ $0.00, p=.99$, and for word items, $F 2(1,23)=0.00, p=.99$. At both SOAs, the output error was identical $(M=1.05)$. Also, the main effect of priming direction was not significant across participants, $F 1(1,29)=0.27, p=.61$, and across word items, $F 2(1,23)$ $=0.00, p=.97$. That is, there was no difference in output error in the forward $(M=1.05)$ and backward $(M=1.04)$ priming directions. However, the prime type main effect was significant for participants, $F 1(1,29)=1895.04, p<.001$, and for word items, $F 2(1,23)=$ $5.59, p<.05$. Lower error rates were found for related word pairs $(M=0.98)$ than for unrelated pairs $(M=1.12)$.

The SOA x Prime interaction was significant for participants, $F 1(1,29)=436.61$, $p<.001$, and word items, $F 2(1,23)=11.10, p<.01$. A significant interaction for SOA x Direction was found for participants, $F 1(1,29)=15.28, p<.01$, but not for word items, $F 2(1,23)=2.87, p=.10$. The interaction for Direction $\times$ Prime was statistically significant for participants, $F 1(1,29)=47.30, p<.001$, but again, not for word items, $F 2(1,23)=0.21, p=.65$. Likewise, the SOA $\mathrm{x}$ Direction $\mathrm{x}$ Prime interaction was statistically significant across participants, $F 1(1,29)=59.60, p<.001$, but not across word items $F 2(1,23)=1.39, p=.25$.

Similar to Moss et al.'s (1994) statistical analysis of interaction effects, only a statistically significant interaction found across both participants and word items was followed up for its simple effects. The simple effects of the SOA x Prime interaction showed that the mean error rate was significantly lower for related words $(M=0.95)$ than for unrelated words $(M=1.14)$ at the short SOA for both participants, $F 1(1,59)=816.99$, 
$p<.001$, and word items, $F 2(1,47)=13.10, p<.01$. At the long SOA, the lower mean error rate for related words $(M=1.00)$ than for unrelated words $(M=1.09)$ was statistically significant for participants, $F 1(1,59)=430.33, p<.001$, but not for word items, $F 2(1,47)=2.04, p=.16$.

The final part of the analysis examined the roles of target word frequency and semantic relatedness in priming. A series of multiple regression analyses were conducted in order to determine the relationship these two variables had with forward and backward priming in the neural network. The bivariate and partial correlations of the two predictors with forward priming at both SOAs are presented in Table 22. At both the short $(r=0.47, p<.05)$ and long $(r=0.50, p<.05)$ SOAs, target word frequency was significantly correlated with the magnitude of forward priming. High frequency target words produced greater magnitudes of forward priming. Table 23 provides the summary of the regression analyses for forward priming at both SOAs. Target word frequency by itself accounted for a significant proportion of variance in the magnitude of forward priming in both the short SOA $\left(R^{2}=0.31\right), F(1,22)=1.22, p<.01$, and long SOA, $\left(R^{2}=\right.$ $0.41), F(1,22)=15.30, p<.01$. The bivariate and partial correlations of the two predictors with backward priming at both SOAs are presented in Table 24. The strongest correlation was found between forward semantic relatedness and backward priming at the long SOA, but it was not statistically significant $(r=-0.47, p<.05)$. Related word pairs with lower semantic overlap produced greater magnitudes of backward priming at the long SOA. None of the other predictor variables were significantly correlated with forward priming at both SOAs. Table 25 provides the summary of the regression analyses for backward priming. Semantic relatedness at the long SOA accounted for the 
largest amount of variance amongst all of the predictor variables, $R^{2}=0.22, F(1,22)=$ $6.17, p<.05$. No other predictor variables were found to account for a statistically significant proportion of the variance for backward at the long SOA.

\section{Discussion}

The performance of the neural network revealed that it is capable of displaying both forward and backward priming at both SOA presentations of the words. However, the presence of backward priming is counterintuitive given how associative relations are formed in an SRNN. This follows the reasoning that the contextual layer of the network forms relations between words based on the order of their presentation, so a backward relation should not have formed. The semantic relatedness between related word items did influence the degree of priming in the backward priming and this might be the reason for backward priming effects. The problem with this explanation is that an SRNN is not influenced by the semantic overlap between related and items as was shown in Moss et al.'s (1994) study. This is because both a prime and target word's output in the semantic layer is completely determined by the input it receives as a result of the interactive processing between the hidden and context layers. Therefore, the semantic output for a prime word has no bearing on the output of the target word.

So why does backward priming occur in the neural network and why do the results show that semantic relatedness has an impact on it? The explanation is that there is a more subtle relationship between the context and hidden layers regarding the semantic relatedness between related words. The first part of the explanation is that when related words shared a greater degree of similarity in their semantic vector patterns, it is more likely that they produce similar hidden layer activation states. Hidden layer 
units are responsible for producing the semantic layer's activation values. Thus, if two words have similar semantic activation patterns, it is likely that they rely upon similar hidden layer activations to produce their respective semantic output. In order to show that this is the case, a cluster plot analysis was conducted on the first simulation's hidden layer states in order compare similarities between related words when they are presented individually. The cluster plot analysis is an established research tool used by neural network researchers for identifying underlying patterns in the processing behaviour of different layers within a neural network (O'Reilly \& Munakata, 2003).

A cluster plot groups more similar items into categories and signifies their degree of similarity by distance length. Vertical "branches" in the analysis represent categorical groupings while horizontal "leaves" denote the similarity distance between items within a particular grouping. The analysis for individual items was identified on the cluster plot by what related pair the word belonged to (e.g., R1), making a distinction as to whether the word is the prime (Prm) or target (Tar) in the backward priming direction and the degree of semantic relatedness between words (High vs. Low). The results in Figure 2 show that 10 related pairs (each identified by an asterisk in Figure 2) were properly grouped into their own unique leaf cluster and that 7 of these pairs had a high semantic relatedness. To contrast these results, another cluster analysis was conducted between the hidden states for the backward target items and their unrelated primes (e.g., U1) when presented individually to the neural network. The results are shown in Figure 3. There was not a single case of a unrelated prime word that was properly paired with its corresponding backward target. 
When two words share similar hidden layer activation states, they will produce similar contextual activation states as well. This is because the context layer's activation state is essentially a copy of the hidden layer's activation state from the previous time step (though degraded somewhat due to the hysteresis factor). In the backward priming direction, this similarity in contextual information facilitates the production of a hidden layer activation state similar to the hidden layer activation state in the forward priming direction. In order to illustrate this, Figure 4 shows the cluster analysis of the context layer state activation for related items when they acted as target words in the forward and backward priming directions. The cluster analysis properly grouped virtually all of the related words with the exception of two pairs (each identified by an asterisk in Figure 4) with low semantic relatedness. Since the hidden and context layer states are the same for target word in both priming directions, the semantic layer activation states will be similar as well. Because related words have a similar semantic vector representation, a similar semantic layer activation state is produced in the backward and forward priming directions. In turn, this facilitates production of the correct semantic output of the target word in the backward priming direction.

The results from the cluster plot analyses provide valuable insight into how backward priming arises in the neural network model. There is a complex relationship existing among the hidden, context, and semantic layers. At the centre of this relationship is the hidden layer. In a backpropagation network, the hidden layer's essential purpose is to develop a set of representations (based on its connection weights) that are "mapping rules" that take the input received from the input layer to produce a specific (and desired) activation pattern in the output layer. Without a hidden layer, the neural network is 
severely limited in its ability to develop effective mapping rules using more complex input-output pattern representations or larger sets of training pattern data.

The hidden layer in a lexical processing neural network is vital because it can handle the arbitrary relationship (similar to natural languages) that exists between the orthographic (or phonological) and semantic pattern representations assigned to a given word. The connection weights between the hidden layer and semantic layer represent semantic information. Likewise, the connection weights between the hidden and context layer represent associative information.

As the cluster plot analysis showed, backward priming results from similar hidden and context layer activation states between related word pairs. The input layer pattern from either word in a semantically related pair will produce similar hidden activation layer states. Similar hidden layer activation states between two semantically related words occur because it is highly probable they rely upon many of the same hiddensemantic layer connection weights to produce their similar semantic layer activation patterns. This hidden layer similarity is also responsible for the forward associative relation's role in backward priming. In this case, similar hidden activation states between two related words produces similar context layer activation states. This allows both words to use similar associative information stored in the connection weights sent from the context layer to the hidden layer. Because either word's input pattern produces a similar hidden activation pattern and the context layer sends similar input to the hidden layer when either word is the target word, the hidden layer's activation state for the target word in either priming direction will also end up being very similar. Altogether, it is the combined interaction of the semantic relatedness and the forward associative relation 
existing between asymmetrically related word pairs that produce backward priming in the neural network.

The results from the SRNN's lexical decision task performance and analysis of its activation states does not provide a complete understanding of the role of semantic relatedness in producing backward priming. The difference between the average number of shared features for related word pairs in the two levels of semantic relatedness may have been too small to produce reliable results. An increase in this difference can be accomplished by using sets of related word pairs with no semantic relatedness rather than low levels of semantic relatedness. The presently held view that semantic relatedness is involved in the neural network's backward priming effects will only be supported if backward priming does not occur for non-semantically related words. However, there are certain difficulties that arise when creating non-semantically related words for the model and these concerns are addressed in the main discussion.

A second factor possibly obscuring the contribution of semantic relatedness is the effect of the forward associative relation on backward priming. During training, all related word pairs had the same probability of co-occurring together in the forward priming direction within a sequence. The reason for not varying this probability between different word pairs was based on the fact that an SRNN forms an association that is sensitive to presentation order. Hence, there was no reason to assume, nor any previous SRNN research to indicate, that a unidirectional (forward) association formed between two input patterns could facilitate the network's performance when the patterns were presented in their non-associated (backward) order. The cluster plot analysis supports the notion that the forward associative relationship plays a role in the model's ability to 
produce backward priming effects, but cannot provide more specific details on how influential the forward associative relationship actually is. Further tests of the model should vary the degree of probability of co-occurrence of related prime and target words in the forward priming direction in order to fully elucidate the contribution of the forward associative relationship towards backward priming.

The results from the cluster plot analysis and the conclusions drawn from these results concerning how backward priming effects arise in the neural network must be interpreted with caution. Cluster plot analyses must be conducted on several training simulations in order to establish the reliability of the present study's cluster plot results. A more detailed analysis comparing related and unrelated word pair activation values and connection weight changes in the hidden and context layers over an entire training phase would also corroborate the present study's explanation of how the SRNN produces backward priming. Based on the present study's interpretation of the cluster plot results, however, the model's ability to elicit backward priming between related words due to a complex interaction between the neural network's associative and semantic mechanisms.

One notable failure of the SRNN's performance was that high frequency target words tended to produce a greater magnitude of forward priming. This contradicts the present study's experimental results, which showed that target word frequency had little effect on the magnitude of forward priming. The method for implementing frequency effects during the SRNN's training phase was similar to the method used by both Plaut (1995) and Plaut and Booth (2000). In those two studies, lower frequency target words produced a greater magnitude of forward priming. Thus, it is necessary to determine why the current neural network failed to replicate results from the human research as well. 
One potential answer is that, during training, high frequency target words have a slightly greater probability of occurring after its related prime word in a sequence. If a prime word is presented, both high and low frequency targets have a 0.25 probability of being selected as the following word. If the related target word is not selected in this way, it can still be selected when the target word is chosen from amongst the entire word set. In this case, a high frequency target word is twice as likely to be selected $(p \cong 0.0166)$ than a low frequency target word $(p \cong 0.0083)$ because word frequency is taken into account. This means that, during the training phase, high frequency related target words actually have a slightly higher probability $(p=0.25+0.0166)$ of being chosen as the target word following the related prime word than does the low frequency related target word $(p=$ $0.25+0.0083$ ). On the other hand, high frequency target words are also twice as likely as low frequency targets to follow an unrelated prime word in a sequence. This should mitigate any benefits from the extra training the high frequency target word was conferred due to its increased co-occurrence with the related prime word. Nonetheless, it is evident that this mitigating effect does not occur based on the relationship found between target word frequency and forward priming effects.

Another potential explanation for the stronger forward priming effects for high frequency target words is that, as a result of the greater occurrence of high frequency target words during training, the neural network's weight connections were adjusted in such a way to allow the neural network to more effectively process high frequency target words following any prime word. Compared to low frequency target words during training, high frequency target words have served as the target word a greater number of times to every word in the word set serving as a prime words. This enables the SRNN to 
more effectively handle a greater variety of contextual information when it is attempting to produce the correct semantic output for high frequency target words. In the forward priming direction, high frequency target words from related word pairs not only benefit from greater exposure to other prime words, but also from its increased co-occurrence with the related prime word. Thus, these high frequency target words produce less error (stronger priming) than low frequency target words. A problem with this account is, both related and unrelated high frequency target words had comparable exposure to a greater variety of prime words during training than their low frequency counterparts. The calculations for priming effects controlled for target word frequency (i.e., high frequency related target words were compared with high frequency unrelated target words). As a result, any potential effects from the high frequency related target word's extra exposure to unrelated prime words during training should be cancelled out when calculating priming effects. Therefore, it is difficult to support this account for the reason why high frequency target words produce greater magnitudes of priming

The two possible accounts put forth explain the target word frequency effect on forward priming as an artifact of the sampling method for target words during the training phase. However, both accounts have weaknesses in their explanations. With this in mind, further tests of the model should also consider implementing alternative sampling procedures for selecting prime and target words during training. Specifically, one sampling procedure should control for the previously mentioned fact that higher frequency related target words have a slightly higher probability of appearing after the related prime word than the low frequency related target word does. This would allow 
the researcher to determine if there is any influence from the high frequency target word's greater exposure to other prime words during training.

To summarize, the SRNN model displayed both forward and backward priming effects at both SOAs. Forward and backward priming effects were similar in magnitude at both SOAs. Backward priming appears to be produced by a complex interaction between the neural network layers in conjunction with the (forward) associative and semantic relationships existing between a related word pair. The reason why higher frequency target words produce greater forward priming still needs to be resolved. The final evaluation of the SRNN's performance requires a comparison with the study's experimental results.

\section{General Discussion}

A comparison between the experimental and simulation results reveals similarities and differences. Both the human participants and the neural network demonstrated priming in both directions and at both SOAs. Human participants produced comparable magnitudes of priming in both directions. However, there were mixed results for the neural network regarding the difference in priming magnitudes in the forward and backward priming directions. At the participant level, forward priming was stronger than backward priming. At the word item level, priming was equivalent in both directions. In backward priming, both the neural network and human participants produced a greater magnitude of priming with lower frequency target words. However, these lexical frequency effects were statistically significant only for human participants. In the forward priming direction, higher frequency target words produced a greater magnitude of priming in the neural network but did not for participants. However, in the 
experiment, higher frequency target words without a backward association strength was positively (but not significantly) correlated with a greater magnitude of forward priming.

The experimental results did not show that semantic relatedness contributed towards forward or backward priming. On the other hand, semantic relatedness played an important role in producing backward priming in the neural network model. This discrepancy in findings between the experimental and model brings into question the cognitive validity of the model. It must be noted that it is important to consider how semantic relatedness was represented in the experiment and the model.

WordNet::Similarity measures semantic relatedness based on synsets shared in common between the prime and target word. A synset can be construed as a means to represent the underlying conceptual or categorical membership of a set of synonymous words. In the neural network, semantic relatedness is defined as semantic feature overlap between the prime and target word. A semantic feature may be construed as a more basic level of semantic representation compared to a synset. Therefore, the difference between the neural network's results and the experimental results may have been due to differing means of representing semantic relatedness. It is also important to note that the neural network's results point towards an interactive process between the forward associative relationship and semantic relatedness being responsible for producing backward priming effects. The results from the experiment could only show that semantic relatedness on its own was not a significant contributor towards backward priming effects. Thus, it is necessary to examine in a future a experiment, the possibility that an interactive relationship exists between forward association strength and semantic relatedness in producing backward priming. 
For asymmetrically related word pairs without a backward association strength and a minimum forward association strength of 0.05 , a stronger forward association strength was associated with a greater magnitude of backward priming for human participants. These results are comparable to the neural network results because none of the word pairs used in the simulation had a backward associative relation. The way in which the neural network uses the forward association to elicit backward priming effects can also provide a model for Seidenberg et al.'s (1984) post-lexical relatedness-search mechanism. As stated earlier, Thompson-Schill et al. (1998) concluded that backward priming in asymmetrically related word pairs arises solely from a word pair's semantic relation. However, results from the current study showed that, for asymmetrically related words with no existing backward association strength, the word pair's forward association strength is a significant predictor of the magnitude of backward priming. It is plausible that both the (forward) associative and semantic mechanisms contribute towards backward priming. MacNamara (2005) has also taken up the position that both associative and semantic features contribute towards backward priming in asymmetrically related word pairs. In the current study, support for this argument comes from the analysis of the neural network's hidden layer activation states while it processes a word pair sequence. In the model, a forward associative relation between related word pairs is necessary in order to produce backward priming. Semantic relatedness mediates the magnitude of backward priming. Whether a semantic relation is also necessary for producing backward priming in the neural network has not been resolved.

In order to properly delineate the role of semantic relatedness in backward priming within the model, future simulations should include related word pairs without 
any semantic overlap (i.e., simulating compound cue words). There are certain problems in creating associated but non-semantically unrelated words for the neural network. Semantic relatedness was defined as the number of active semantic features shared between related words. The shared number of inactive semantic features was not taken into consideration, even though there is little distinction between active and inactive features by the neural network in terms of what they signify or the means by which they are produced. In essence, an inactive semantic unit shared by two words also constitutes a semantic feature overlap. Considering the fact that most semantic units were inactive for the desired semantic representation of the words used for training and testing in the model, there is potentially (and technically) a large degree of semantic overlap shared between any two words from the word set. This would be a problem for simulating priming effects for compound cue word pairs since they share no semantic relation.

A potential way to resolve this problem is to have the prime and target words have inverse desired patterns of active-inactive semantic features between them resulting in no semantic overlap between them. However, a similar method would have to be adopted for the unrelated "filler" words as well since they do not share a semantic relation with any other word within the word set. Generating a word set of compound cue words and unrelated filler words that meets these requirements is either not possible or extremely difficult to do at this point. Needless to say, a future study of the neural network simulating compound cue words is necessary in order to lend further support to its validity as a model of human lexical processing.

These findings and conclusions drawn from the neural network results can provide a possible explanation of what occurs within the human lexical processing 
system during semantic priming. The SRNN findings indicates that both the associative and semantic relationship between a word pair contribute towards backward priming. In accordance with Moss et al's (1995) study on the "associative boost" effect on priming for associative/semantically related items, there are separate mechanisms for semantic and associative information responsible for backward priming. The neural network's results go further in its claims, though, by demonstrating that the model's associative and semantic mechanisms produce backward priming through an interactive process rather than by separate contributions.

When drawing conclusions about the role of associative relatedness in semantic priming, the current study's finding of the forward association strength role in backward priming must be interpreted with caution. Forward association was not a good predictor of forward priming in all related word pairs. Furthermore, the current study was unable to analyze the effects that backward association strength had on backward priming. In light of these issues, association strength might not be a suitable measure of the associative relationship existing between related words. Any future studies of backward priming might consider using alternative measures of a related word pair's associative relation. One approach, posited by MacNamara (2005) and Hutchinson (2003), is basing the strength of the associative relation between related words on the number of target words that a prime word has associated with it. Virtually all prime words used in the current study have more than one associated target word listed in Neslon et al.'s (1998) word association norms. In the case of the prime word having multiple associated target words, the target word being presented might have to compete with the other target words for the dominant activation of its lexical representation. Subsequently, the greater the 
number of words associated with a prime word, the slower recognition will be for the target word.

Related to the issue of multiple target associates is the rank of the target word amongst the multiple associates of the prime word. A target word that has the strongest association strength with the prime word is also ranked as the prime word's primary associate. Anaki and Henik (2003) found that the primary target word associate for a prime word usually produces the greatest magnitude of priming. Also, the conventional association strength measure has no relation to the magnitude of priming when comparing primary associates with one another. Anaki and Henik have also argued that the associative primacy of the target word has been a confounding variable in previous semantic priming examining the influence of association strength on priming. This is because a target word with a stronger association strength is also more likely to be the primary associate of a prime word.

Both of these alternative measures of the associative relation between a related word pair are worth investigating in future research. Before they can be implemented, however, it is important to consider the possibility that association strength and these other two measures are all involved to one degree or another in representing the associative relationship between a related word pair. If this is the case, then a valid measure of the associative relation between related words requires a combined measure of these three existing measures. Otherwise, any semantic priming study that examines the role of associative relatedness will be based on an inaccurate measure of word association. 
One future research goal for the SRNN model should be the implementation of longer word sequences during training. This type of training would lend a greater degree of validity to the model because the neural network's training regimen would be closer to processing actual sentences rather than just word pairs. If human beings mainly acquire associative relations between words from their co-occurrence within sentences, then it is important to see if an SRNN can do the same. A parallel concern was raised by Moss et al. (1994) regarding their model's training method in relation to the actual co-occurrence of associated prime and target words within textual corpora. When studying the actual co-occurrence of related words within a linguistic context, they tend to be separated by a number of intervening words. Rapp and Wettler (1991) found the strongest correlations between the co-occurrence of related words when they are separated by up to 12 intervening words within a textual corpora database. It is possible to train an SRNN using word sequences longer than two words during training by adjusting the hysteresis value, but it is unknown whether it can effectively develop associations between words using word sequences of this size. Consequently, the neural network's capability to display priming under similar conditions is an important issue to investigate in future studies.

The neural network used in the current study shows a great deal of promise in providing insight into how backward priming is formed and elicited in the human lexical processing system. The discrepancies between the neural network's performance and human results regarding target word frequency effects on priming and directional priming asymmetry need to be resolved in future study of the model. In spite of this, the neural network model does provide a plausible account of how backward priming arises in 
asymmetrically related word pairs. In order to validate this account of backward priming, further study is needed to determine the contributions of associative and semantic features towards priming effects produced by human beings. 


\section{References}

Anaki, D., \& Henik, A. (2003). Is there a "strength effect" in automatic semantic priming? Memory \& Cognition, 31(2), 262-272.

Becker, C.A. (1979). Semantic context and word frequency effects in visual word recognition. Journal of Experimental Psychology Human Perception and Performance, 5, 252-259.

Becker, S., Moscovitch, M. Behrmann, M. \& Joordens, S. (1997). Long-term semantic priming: A computational account and empirical evidence. Journal of Experimental Psychology: Learning, Memory and Cognition, 23(5), 1059-1082.

Bentin, S., McCarthy, G., \& Wood, C. C. (1985). Event-related potentials, lexical decision, and semantic priming. Electroencephalography and Clinical Neurophysiology, 60, 343-355.

Chiarello, C., Burgess, C., Richards, L., \& Pollock, A. (1990). Semantic and associative priming in the cerebral hemispheres: Some words do, some words don't . . . sometimes, some places. Brain and Language, 38, 75-104.

Chwilla, D.J., Hagoort, P., \& Brown, C.M. (1998). The mechanism underlying backward priming in a lexical decision task: Spreading activation versus semantic matching. Quarterly Journal of Experimental Psychology, 51A, 531-560.

Cree, G. S., McRae, K., \& McNorgan, C. (1999). An attractor model of lexical conceptual processing: Simulating semantic priming. Cognitive Science, 23, 371414.

De Groot, A. M. B. (1984). Primed lexical decision: Combined effects of the proportion of related prime-target pairs and the stimulus-onset asynchrony of prime and 
target. Quarterly Journal of Experimental Psychology, 36A, 253-280.

De Groot, A. M. B. (1985). Word-context effects in word naming and lexical decision. Quarterly Journal of Experimental Psychology, 37A, 281-297.

den Heyer, K., Goring, A. \& Dannenbring, G.L. (1985). Semantic priming and word repetition: The two effects are additive. Journal of Memory and Language, 24, 699-716.

Durgunoglu, A.Y., \& Neely, J.H. (1987). On obtaining episodic priming in a lexical decision task following paired-associate learning. Journal of Experimental Psychology: Learning, Memory and Cognition, 13, 206-222.

Elman, L.J. (1990). Finding structure in time. Cognitive Science, 14, 179-211.

E-Prime (Version 1.2). (2000). Pittsburgh, PA: Psychology Software Tools, Inc.

Fischler, I. (1977). Semantic facilitation without association in a lexical decision task. Memory \& Cognition, 5, 335-339.

Fodor, J. (1983). The Modularity of Mind. Cambridge, MA: MIT Press.

Forbach, G. B., Stanners, R. F., \& Hochhaus, L. (1974). Repetition and practice effects in a lexical decision task. Memory and Cognition, 2, 337-339.

Hagoort P., Brown, C. \& Swaab, T.Y. (1996). Lexical-semantic event-related potential effects in patients with left hemisphere lesions and aphasia, and patients with right hemisphere lesions without aphașia. Brain, 119, 627-649.

Hutchinson, K.A. (2002). The effect of asymmetrical association on positive and negative semantic priming. Memory \& Cognition, 30, 1263-1276.

Hutchison, K. A. (2003). Is semantic priming due to association strength or feature overlap? A micro-analytic review. Psychonomic Bulletin and Review, 10(4), 785- 
813.

Jordan, M.I. (1986). Attractor dynamics and parallelism in a connectionist sequential machine. Proceedings of the Eighth Annual Conference of the Cognitive Science Society, USA, 8, 531-546.

Kahan, T.A., Neely, J.H., \& Forsythe, W.J. (1999). Dissociated backward. priming effects in lexical decision and pronunciation tasks. Psychonomic Bulletin \& Review, 6(1), 105-110.

Koivisto, M., \& Revonsuo, A. (2001). Cognitive representations underlying the N400 priming effect. Cognitive Brain Research 12: 487-490.

Koriat, A. (1981). Semantic facilitation in lexical decision as a function of prime-target association. Memory \& Cognition, 9, 587-598.

Kucera, H. \& Francis, W.N. (1967). Computational analysis of present-day American English. Providence, RI: Brown University Press.

Lucas, M. (2000). Semantic priming without association: A meta-analytic review. Psychnomic Bulletin \& Review, 7(4), 618-630.

Marr, D. (1982). Vision. San Francisco: W. H. Freeman.

Masson, M.J. (1995). A distributed memory model for semantic priming. Journal of Experimental Psychology: Learning, Memory \& Cognition, 21, 3-23.

Matlab (Version 7.0). (2004). Natick, MA: The Mathworks, Inc.

McNamara, T. P. (2005). Semantic priming: Perspectives from memory and word recognition. New York: Psychology Press.

McRae, K., \& Boisvert, S. (1998). Automatic semantic similarity priming. Journal of Experimental Psychology: Learning, Memory, and Cognition, 24, 558-572. 
McCrae, K., De Sa, V.R., \& Seidenberg, M.S. (1997). On the nature and scope of featural representations of word meaning. Journal of Experimental Psychology: General, $126,99-130$.

Miller, G.A., Fellbaum, C., Tengi, R., Wakefield, P., Poddar, R., Langone, H., \& Haskell, B. (2006). WordNet: A Lexical Database for the English Language. http://wordnet.princeton.edu/

Moss, H. E., Hare, M. L., Day, P., \& Tyler, L. K. (1994). A distributed memory model of the associative boost in semantic priming. Connection Science, 6(4), 413-427.

Moss, H. E., Ostrin, R. K., Tyler, L. K., \& Marslen-Wilson, W. D. (1995). Accessing different types of lexical semantic information: Evidence from priming. Journal of Experimental Psychology: Learning, Memory, and Cognition, 21(4), 863-883.

Neely, J. H. (1991). Semantic priming effects in visual word recognition: A selective review of current findings and theories. In D. Besner, \& G. W. Humphreys (Eds.), Basic processes in reading (pp. 264-336). Hillsdale, NJ: Lawrence Erlbaum Associates.

Nelson, D. L., McEvoy, C. L., \& Schreiber, T. A. (1998). The University of South Florida word association, rhyme, and word fragment norms. http://www.usf.edu/FreeAssociation/.

O'Reilly, R. C. \& Munakata, Y. (2000). Computational Explorations in Cognitive Neuroscience: Understanding the Mind by Simulating the Brain. Cambridge, MA: MIT Press.

O'Reilly, R.C., Dawson, C.K., \& McClelland, J.L. (2003). PDP++ Mac OSX Version 3.2 [Computer software and manual]. Retrieved from 
ftp://grey.colorado.edu/pub/oreilly/pdp++/

Patwardhan, S. \& Pedersen, T. (2006). Using WordNet based context vectors to estimate the semantic relatedness of concepts. Proceedings of the EACL 2006 Workshop. Italy, 1-8.

Pedersen, T., Patwardhan, S., \& Michelizzi, J. (2004). WordNet::Similarity - Measuring the relatedness of concepts. Proceedings of the Fifth Annual Meeting of the North American Chapter of the Association for Computational Linguistics, USA, 5, 3841.

Perea, M., \& Gotor, A. (1997). Associative and semantic priming effects occur at very short stimulus-onset asynchronies in lexical decision and naming. Cognition, 62, 223-240.

Peterson, R. R. \& Simpson, G. B. (1989). The effect of backward priming on word recognition in single-word and sentence contexts. Journal of Experimental Psychology: Learning, Memory, and Cognition, 15, 1020-1032.

Plaut, D. C. (1995). Semantic and associative priming in a distributed attractor network. Proceedings of the 17th Annual Conference of the Cognitive Science Society, USA, 17, 37-42.

Plaut, D. C. \& Booth, J. R. (2000). Individual and developmental differences in semantic priming: Empirical and computational support for a single-mechanism account of lexical processing. Psychological Review, 107, 786-823.

Rapp, R. \& Wettler, M. (1991). Prediction of free word associations based on Hebbian learning. Proceedings of the International Joint Conference on Neural Networks (pp. 25-29), Singapore, Vol.1. 
Resnik, P. (1993). Selection and information: A class-based approach to lexical relationships. Unpublished doctoral dissertation, University of Pennsylvania, Philadelphia.

Schreuder R., Flores d' Arcais, G., Glazenburg, G. (1984) Effects of perceptual and conceptual similarity in semantic priming. Psychological Research. (45), 339354.

Seidenberg, M. S., Waters, G. S., Sanders, M., \& Langer, P. (1984). Pre- and postlexical loci of contextual effects on word recognition. Memory and Cognition, 12, 315328.

Shelton, J.R., \& Martin, R.C. (1992). How semantic is automatic semantic priming? Journal of Experimental Psychology: Learning, Memory, and Cognition, 18, 1191-1210.

Smolensky, P. (1995). On the proper treatment of connectionism. In C. Macdonald \& G. Macdonald (Eds.), Connectionism: Debates on psychological explanation, Volume 2 (pp.28-89). Oxford, UK: Basil Blackwell.

Stone, G. O., \& van Orden, G. C. (1992). Resolving empirical inconsistencies concerning priming, frequency, and nonword foils in lexical decision. Language \& Speech, $35,295-324$.

Thompson-Schill, S. L., Kurtz, K. J., \& Gabrieli, J. D. E. (1998). Effects of semantic and associative relatedness on automatic priming. Journal of Memory and Language, $38,440-458$.

van Gelder, T. \& Port, R. (1995). It's about time. In R. Port and T. van Gelder (Eds.), Mind as Motion: Explorations in the Dynamics of Cognition (pp. 1-44). 
Cambridge, MA: MIT Press. 
Table 1

Forward and Backward Priming Results (In Milliseconds) From Previous Lexical Decision Task Studies Using Asymmetrically Associated Words

\begin{tabular}{|c|c|c|c|c|c|}
\hline Study & $\begin{array}{l}\text { Forward } \\
\text { Priming }\end{array}$ & $\begin{array}{c}\text { Backward } \\
\text { Priming } \\
\end{array}$ & SOA & $\begin{array}{c}\text { Relatedness } \\
\text { Proportion }\end{array}$ & $\begin{array}{c}\text { Non-Word } \\
\text { Ratio }\end{array}$ \\
\hline Koriat & 34 & 35 & 650 & .50 & .67 \\
\hline Peterson 1 & & 22 & 0 & .50 & .67 \\
\hline Peterson 2 & & 26 & 200 & .50 & .67 \\
\hline Peterson 3 & 40 & 37 & 500 & .50 & .67 \\
\hline Chwilla & 61 & 27 & $0 / 500^{\mathrm{a}}$ & .75 & .80 \\
\hline $\begin{array}{l}\text { Thompson- } \\
\text { Schill } 1\end{array}$ & 24 & 17 & 250 & .50 & .67 \\
\hline $\begin{array}{l}\text { Thompson- } \\
\text { Schill } 2\end{array}$ & 25 & 17 & 250 & .36 & .60 \\
\hline Kahan 1 & & 30 & 150 & .80 & .875 \\
\hline Kahan 2 & & 26 & 500 & .80 & .875 \\
\hline Hutchinson $^{\mathrm{b}}$ & 36 & -4 & 700 & .50 & .50 \\
\hline
\end{tabular}

Notes. Citations are Koriat, 1981; Peterson \& Simpson, 1989; Chwilla, Hagoort, \& Brown, 1998; Kahan, Neely, \& Forsythe, 1998; Thompson-Schill, Kurtz, \& Gabrieli, 1998; and Hutchinson, 2002. In Peterson \& Simpson's (1989) study, related words were not distinguished between compound-cues and asymmetric associates.

${ }^{a}$ Lexical decision task had half of its trials with an SOA of 0 and the remainder with an SOA of 500.

${ }^{b}$ Continuous lexical decision task: Does not involve sequences of prime/target word pairs. Instead, the participant responds to every word and non-word item presented in sequence (SOA = interval between items). 
Table 2

Forward and Backward Priming Results (In Milliseconds) From Previous Lexical Decision Task Studies Using Compound Cue Words

\begin{tabular}{lccccc}
\hline Study & $\begin{array}{l}\text { Forward } \\
\text { Priming }\end{array}$ & $\begin{array}{c}\text { Backward } \\
\text { Priming }\end{array}$ & SOA & $\begin{array}{c}\text { Relatedness } \\
\text { Proportion }\end{array}$ & $\begin{array}{c}\text { Non-Word } \\
\text { Ratio }\end{array}$ \\
\hline Seidenberg & 27 & 21 & 500 & .50 & .67 \\
Shelton $1^{\text {a }}$ & 38 & 19 & 750 & .24 & .45 \\
Shelton 2 & 32 & 25 & 750 & .76 & .72 \\
Chwilla & 25 & 29 & $0 / 500^{\mathrm{b}}$ & .75 & .80 \\
Kahan 1 & 26 & 150 & .80 & .875 \\
\\
Kahan 2
\end{tabular}


Table 3

The set of asymmetrically related word pairs used in the study with corresponding association strength and lexical frequency values $(N=55)$

\begin{tabular}{|c|c|c|c|c|c|c|}
\hline \multicolumn{2}{|c|}{$\begin{array}{l}\text { Word Pair } \\
\text { (Forward Direction) }\end{array}$} & \multicolumn{2}{|c|}{ Association Strength } & \multirow[t]{2}{*}{$\begin{array}{c}\text { Semantic } \\
\text { Relatedness }\end{array}$} & \multicolumn{2}{|c|}{$\begin{array}{c}\text { Lexical } \\
\text { Frequency }\end{array}$} \\
\hline Prime & Target & Forward & Backward & & Prime & Target \\
\hline crown & king & 0.47 & 0.01 & 0.26 & 19 & 88 \\
\hline lizard & snake & 0.15 & 0.02 & 0.53 & 1 & 44 \\
\hline ocean & water & 0.22 & 0.02 & 0.47 & 34 & 442 \\
\hline path & road & 0.23 & 0.03 & 0.40 & 44 & 197 \\
\hline sheep & wool & 0.29 & 0.21 & 0.45 & 23 & 10 \\
\hline lamp & light & 0.77 & 0.02 & 0.48 & 18 & 333 \\
\hline eagle & bird & 0.48 & 0.05 & 0.44 & 5 & 31 \\
\hline comb & brush & 0.63 & 0.16 & 0.55 & 6 & 44 \\
\hline mold & bread & 0.12 & 0.00 & 0.10 & 45 & 41 \\
\hline town & city & 0.52 & 0.31 & 0.89 & 212 & 393 \\
\hline scissors & cut & 0.87 & 0.03 & 0.42 & 1 & 192 \\
\hline mint & candy & 0.23 & 0.00 & 0.49 & 7 & 16 \\
\hline engine & car & 0.35 & 0.01 & 0.41 & 50 & 274 \\
\hline flea & $\operatorname{dog}$ & 0.34 & 0.00 & 0.24 & 2 & 75 \\
\hline mud & dirt & 0.59 & 0.28 & 0.44 & 32 & 43 \\
\hline bar & drink & 0.39 & 0.00 & 0.25 & 82 & 82 \\
\hline inch & foot & 0.47 & 0.02 & 0.53 & 40 & 70 \\
\hline stem & flower & 0.43 & 0.01 & 0.45 & 29 & 23 \\
\hline cottage & house & 0.38 & 0.00 & 0.30 & 19 & 591 \\
\hline sword & knife & 0.30 & 0.05 & 0.47 & 7 & 76 \\
\hline crater & moon & 0.39 & 0.00 & 0.06 & 2 & 60 \\
\hline crew & ship & 0.19 & 0.00 & 0.25 & 36 & 83 \\
\hline appear & see & 0.15 & 0.00 & 0.34 & 118 & 772 \\
\hline tobacco & smoke & 0.33 & 0.00 & 0.64 & 19 & 41 \\
\hline train & track & 0.32 & 0.17 & 0.39 & 82 & 38 \\
\hline brandy & wine & 0.15 & 0.00 & 0.62 & 7 & 72 \\
\hline beaver & dam & 0.27 & 0.15 & 0.36 & 2 & 5 \\
\hline dentist & pain & 0.08 & 0.00 & 0.29 & 12 & 88 \\
\hline
\end{tabular}


Table 3 (continued)

The set of asymmetrically related word pairs to be used in the study with corresponding association strength and lexical frequency values $(N=55)$

\begin{tabular}{|c|c|c|c|c|c|c|}
\hline \multicolumn{2}{|c|}{$\begin{array}{l}\text { Word Pair } \\
\text { (Forward Direction) }\end{array}$} & \multicolumn{2}{|c|}{ Association Strength } & \multirow[t]{2}{*}{$\begin{array}{c}\text { Semantic } \\
\text { Relatedness } \\
\end{array}$} & \multicolumn{2}{|c|}{$\begin{array}{l}\text { Lexical } \\
\text { Frequency }\end{array}$} \\
\hline Prime & Target & Forward & Backward & & Prime & Target \\
\hline sheet & paper & 0.42 & 0.01 & 0.43 & 45 & 157 \\
\hline penguin & tuxedo & 0.13 & 0.04 & 0.03 & 0 & 0 \\
\hline indian & feather & 0.06 & 0.00 & 0.18 & 52 & 6 \\
\hline piano & key & 0.12 & 0.00 & 0.45 & 38 & 88 \\
\hline onion & cry & 0.20 & 0.00 & 0.09 & 15 & 48 \\
\hline interest & money & 0.06 & 0.00 & 0.20 & 330 & 265 \\
\hline pigeon & statue & 0.04 & 0.00 & 0.10 & 3 & 15 \\
\hline picnic & ant & 0.17 & 0.03 & 0.09 & 15 & 6 \\
\hline factory & plant & 0.05 & 0.00 & 0.50 & 32 & 125 \\
\hline dream & sleep & 0.25 & 0.19 & 0.17 & 64 & 65 \\
\hline salt & pepper & 0.70 & 0.69 & 0.12 & 46 & 13 \\
\hline silver & gold & 0.63 & 0.47 & 0.70 & 29 & 52 \\
\hline coffee & tea & 0.44 & 0.36 & 0.69 & 78 & 28 \\
\hline iron & steel & 0.25 & 0.22 & 0.71 & 43 & 45 \\
\hline fingers & hands & 0.22 & 0.14 & 0.47 & 66 & 289 \\
\hline image & mirror & 0.45 & 0.27 & 0.46 & 27 & 119 \\
\hline termite & wood & 0.30 & 0.00 & 0.26 & 0 & 55 \\
\hline canyon & deep & 0.04 & 0.00 & 0.35 & 12 & 109 \\
\hline zebra & stripe & 0.45 & 0.05 & 0.14 & 1 & 4 \\
\hline maze & lost & 0.07 & 0.00 & 0.09 & 6 & 173 \\
\hline duck & water & 0.02 & 0.00 & 0.29 & 9 & 442 \\
\hline stove & hot & 0.28 & 0.00 & 0.43 & 8 & 187 \\
\hline chip & shoulder & 0.04 & 0.00 & 0.11 & 17 & 61 \\
\hline fan & air & 0.23 & 0.00 & 0.39 & 18 & 257 \\
\hline towel & dry & 0.28 & 0.02 & 0.14 & 6 & 68 \\
\hline garlic & smell & 0.13 & 0.00 & 0.39 & 4 & 34 \\
\hline afraid & run & 0.01 & 0.00 & 0.20 & 57 & 212 \\
\hline
\end{tabular}


Table 4

Example of a Base Set With The Eight Possible Experimental Conditions For The Related Word Pair "KING $\rightarrow$ CROWN"

Priming Direction

\begin{tabular}{lccccc}
\cline { 5 - 6 } & \multicolumn{2}{c}{ Forward } & & \multicolumn{2}{c}{ Backward } \\
\cline { 2 - 3 } \cline { 5 - 6 } Condition & Word & Non-Word & & Word & Non-Word \\
\hline \multirow{2}{*}{ Related } & CROWN-KING & CROWN-FINT & KING-CROWN & KING-TROWN \\
Unrelated & RIVER-KING & RIVER-FINT & & FIND-CROWN & FIND-TROWN \\
\hline
\end{tabular}


Table 5

Mean Reaction Times and Priming Results

From the Experiment

\begin{tabular}{lcc}
\hline & \multicolumn{2}{c}{ Priming Direction } \\
\cline { 2 - 3 } Prime Type & Forward & Backward \\
\hline & \multicolumn{2}{c}{ Short SOA } \\
Related & $630(168)$ & $666(178)$ \\
Unrelated & $648(172)$ & $684(195)$ \\
Priming & 18 & 18 \\
\hline & \multicolumn{1}{c}{ Long SOA } \\
Related & $577(162)$ & $611(185)$ \\
Unrelated & $594(179)$ & $624(178)$ \\
Priming & 17 & 13 \\
\hline $\begin{array}{l}\text { Note. Values enclosed in parentheses represent } \\
\text { standard deviation values. }\end{array}$
\end{tabular}


Table 6

Overall Percentage of Errors Made In Each

Priming Condition

\begin{tabular}{lcc}
\hline & \multicolumn{2}{c}{ Priming Direction } \\
\cline { 2 - 3 } Prime Type & Forward & Backward \\
\hline \multirow{2}{*}{ Short SOA } \\
Related & 0.5 & 4.1 \\
Unrelated & 2.3 & 5.6 \\
\hline & \multicolumn{2}{c}{ Long SOA } \\
Related & 1.9 & 4.3 \\
Unrelated & 3.3 & 5.6 \\
\hline
\end{tabular}


Table 7

Analysis of Variance for Participant Mean Decision Latencies

\begin{tabular}{|c|c|c|c|c|}
\hline Source & $d f$ & $F$ & $\eta^{2}$ & $p$ \\
\hline & \multicolumn{4}{|c|}{ Between subjects } \\
\hline SOA (S) & 1 & $7.18 * *$ & .08 & .009 \\
\hline \multirow[t]{2}{*}{ S within-group error } & 79 & $(6978.37)$ & & \\
\hline & \multicolumn{4}{|c|}{ Within subjects } \\
\hline Block (B) & 1 & $4.42^{*}$ & .05 & .04 \\
\hline Direction (D) & 1 & $52.50 * * *$ & .40 & .000 \\
\hline Prime $(\mathrm{P})$ & 1 & $17.23 * *$ & .18 & .000 \\
\hline$B \times S$ & 1 & 0.91 & .01 & .34 \\
\hline $\mathrm{B} \times \mathrm{D}$ & 1 & 0.51 & .01 & .48 \\
\hline $\mathrm{B} \times \mathrm{P}$ & 1 & 0.16 & .002 & .69 \\
\hline $\mathrm{D} \times \mathrm{S}$ & 1 & 0.24 & .003 & .62 \\
\hline $\mathrm{D} \times \mathrm{P}$ & 1 & 0.21 & .003 & .65 \\
\hline $\mathrm{P} \times \mathrm{S}$ & 1 & 0.001 & .000 & .98 \\
\hline $\mathrm{B} \times \mathrm{S} \times \mathrm{D}$ & 1 & $6.18^{*}$ & .07 & .02 \\
\hline $\mathrm{B} \times \mathrm{S} \times \mathrm{P}$ & 1 & 0.06 & .001 & .81 \\
\hline $\mathrm{B} \times \mathrm{D} \times \mathrm{P}$ & 1 & 0.25 & .003 & .62 \\
\hline $\mathrm{D} \times \mathrm{S} \times \mathrm{P}$ & 1 & 0.06 & .001 & .80 \\
\hline $\mathrm{B} \times \mathrm{S} \times \mathrm{D} \times \mathrm{P}$ & 1 & 3.51 & .04 & .07 \\
\hline B within-group error & 79 & $(4019.79)$ & & \\
\hline
\end{tabular}

Note. Values enclosed within parentheses represent mean square errors.

${ }^{*} p<.05 .{ }^{* *} p<.01 .{ }^{* * *} p<.001$. 
Table 8

Analysis of Variance for Word Item Mean Decision Latencies

\begin{tabular}{|c|c|c|c|c|}
\hline Source & $d f$ & $F$ & $\eta^{2}$ & $p$ \\
\hline & \multicolumn{4}{|c|}{ Within subjects } \\
\hline SOA (S) & 1 & $221.26^{* *}$ & .80 & .000 \\
\hline Block (B) & 1 & $9.70^{*}$ & .15 & .003 \\
\hline Direction (D) & 1 & $21.96 * *$ & .29 & .000 \\
\hline Prime (P) & 1 & $21.57 * *$ & .29 & .000 \\
\hline $\mathrm{S} \times \mathrm{B}$ & 1 & 1.40 & .03 & .24 \\
\hline $\mathrm{S} \times \mathrm{D}$ & 1 & 0.50 & .01 & .48 \\
\hline $\mathrm{S} \times \mathrm{P}$ & 1 & 0.50 & .01 & .48 \\
\hline $\mathrm{B} \times \mathrm{D}$ & 1 & 0.11 & .002 & .75 \\
\hline $\mathrm{B} \times \mathrm{P}$ & 1 & 0.54 & .01 & .47 \\
\hline $\mathrm{D} \times \mathrm{P}$ & 1 & 0.07 & .001 & .79 \\
\hline $\mathrm{S} \times \mathrm{B} \times \mathrm{D}$ & 1 & 3.70 & .06 & .06 \\
\hline $\mathrm{S} \times \mathrm{B} \times \mathrm{P}$ & 1 & 0.26 & .01 & .61 \\
\hline $\mathrm{S} \times \mathrm{D} \times \mathrm{P}$ & 1 & 0.01 & .000 & .93 \\
\hline $\mathrm{B} \times \mathrm{D} \times \mathrm{P}$ & 1 & 0.39 & .01 & .54 \\
\hline $\mathrm{S} \times \mathrm{B} \times \mathrm{D} \times \mathrm{P}$ & 1 & 3.13 & .06 & .08 \\
\hline $\begin{array}{l}\mathrm{S} \times \mathrm{B} \times \mathrm{P} \\
\text { within-group error }\end{array}$ & 54 & $(4510.27)$ & & \\
\hline
\end{tabular}

Note. Values enclosed within parentheses represent mean square errors.

${ }^{*} p<.01 .{ }^{* *} p<.001$. 
Table 9

Analysis of Variance for Participant Mean Error Percentages

\begin{tabular}{|c|c|c|c|c|}
\hline Source & $d f$ & $F$ & $\eta^{2}$ & $p$ \\
\hline & \multicolumn{4}{|c|}{ Between subjects } \\
\hline SOA (S) & 1 & 1.18 & .02 & .28 \\
\hline \multirow[t]{2}{*}{$\begin{array}{l}S \text { within-group } \\
\text { error }\end{array}$} & 79 & $(19.16)$ & & \\
\hline & \multicolumn{4}{|c|}{ Within subjects } \\
\hline Direction (D) & 1 & $8.42^{*}$ & .10 & .005 \\
\hline Prime $(\mathrm{P})$ & 1 & $21.98 * *$ & .22 & .000 \\
\hline $\mathrm{D} \times \mathrm{S}$ & 1 & 0.01 & .000 & .94 \\
\hline $\mathrm{D} \times \mathrm{P}$ & 1 & 0.01 & .000 & .93 \\
\hline $\mathrm{P} \times \mathrm{S}$ & 1 & 0.14 & .002 & .71 \\
\hline $\mathrm{D} \times \mathrm{S} \times \mathrm{P}$ & 1 & 0.01 & .000 & .95 \\
\hline $\begin{array}{l}\text { D within-group } \\
\text { error }\end{array}$ & 79 & $(24.31)$ & & \\
\hline
\end{tabular}

Note. Values enclosed within parentheses represent mean square errors. ${ }^{*} p<.01 .{ }^{* *} p<.001$. 
Table 10

Analysis of Variance for Word Item Mean Error Percentages

\begin{tabular}{lcccc}
\hline \multicolumn{1}{c}{ Source } & $d f$ & $F$ & $\eta^{2}$ & $p$ \\
\hline & & \multicolumn{4}{c}{ Within subjects } \\
SOA (S) & 1 & 1.42 & .03 & .24 \\
Direction (D) & 1 & $7.20^{*}$ & .12 & .01 \\
Prime (P) & 1 & $13.32^{* *}$ & .20 & .001 \\
S $\times$ D & 1 & 0.11 & .002 & .74 \\
S $\times$ P & 1 & 0.11 & .002 & .74 \\
D $\times$ P & 1 & 0.002 & .000 & .88 \\
S $\times$ D $\times$ P & 1 & 0.004 & .000 & .95 \\
D within-group & & & & \\
$\quad$ error & 54 & $(46.42)$ & & \\
\hline
\end{tabular}

Note. Values enclosed within parentheses represent mean square errors.

${ }^{*} p<.05$. ${ }^{* *} p<.01$. 
Table 11

Bivariate and Partial Correlations of the Predictors with Forward Priming $(N=55)$

\begin{tabular}{lccc}
\hline & $\begin{array}{c}\text { Correlation between each } \\
\text { predictor and forward } \\
\text { priming }\end{array}$ & $\begin{array}{c}\text { Correlation between each } \\
\text { predictor and forward priming } \\
\text { controlling for other predictor }\end{array}$ \\
\hline Predictor & -.04 & -.05 \\
Target frequency & .17 & .17 \\
Forward association & .01 & -.02 \\
Semantic relatedness & & Long SOA & \\
\hline & -.03 & -.05 \\
Target frequency & .12 & .13 \\
Forward association & -.07 & -.09 \\
Semantic relatedness & & & \\
\hline
\end{tabular}


Table 12

Summary of the Multiple Regression Analysis for Variables Predicting the Magnitude of Forward Priming $(N=55)$

\begin{tabular}{lccccc}
\hline & $B$ & $S E B$ & $\beta$ & $R$ & $R^{2}$ \\
\hline & & \multicolumn{5}{c}{ Short SOA } \\
Target frequency & -3.89 & 11.74 & -.05 & .04 & .002 \\
Forward association & 41.19 & 33.80 & .17 & .17 & .03 \\
Semantic Relatedness & -3.70 & 35.53 & -.02 & .01 & .001 \\
All & & & & .17 & .03 \\
\hline & & & Long SOA & & \\
Target frequency & -3.89 & 12.23 & -.04 & .03 & .001 \\
Forward association & 32.63 & 35.07 & .13 & .12 & .01 \\
Semantic Relatedness & -24.25 & 36.86 & -.09 & .07 & .01 \\
All & & & & .15 & .02 \\
\hline
\end{tabular}


Table 13

Bivariate and Partial Correlations of the Predictors with Backward Priming $(N=55)$

\begin{tabular}{|c|c|c|}
\hline Predictor & $\begin{array}{c}\text { Correlation between } \\
\text { each predictor and } \\
\text { backward priming }\end{array}$ & $\begin{array}{c}\text { Correlation between each } \\
\text { predictor and backward } \\
\text { priming controlling for other } \\
\text { predictor }\end{array}$ \\
\hline & \multicolumn{2}{|c|}{ Short SOA } \\
\hline Target frequency & -.19 & -.18 \\
\hline Forward association & .14 & .14 \\
\hline \multirow[t]{2}{*}{ Semantic relatedness } & -.06 & -.06 \\
\hline & \multicolumn{2}{|c|}{ Long SOA } \\
\hline Target frequency & $-.27^{*}$ & -.26 \\
\hline Forward association & .004 & .01 \\
\hline Semantic relatedness & -.11 & -.10 \\
\hline
\end{tabular}

${ }^{*} p<.05$. 
Table 14

Summary of the Multiple Regression Analysis for Variables Predicting the Magnitude of Backward Priming $(N=55)$

\begin{tabular}{lccccc}
\hline & $B$ & $S E B$ & $\beta$ & $R$ & $R^{2}$ \\
\hline Target frequency & -21.67 & 16.37 & -.18 & .19 &, 04 \\
Forward association & 47.40 & 46.59 & .14 & .14 & .02 \\
Semantic Relatedness & -22.49 & 48.86 & -.06 & .06 & .003 \\
All & & & & .24 & .06 \\
\hline & & & & & \\
& & & & & \\
Target frequency & -21.51 & 11.11 & -.26 & .27. & $.07 *$ \\
Forward association & 2.39 & 31.60 & .01 & .004 & .001 \\
Semantic Relatedness & -22.62 & 33.18 & -.09 & .11 & .01 \\
All & & & & .28 & .08 \\
\hline * $p<.05$. & & & & &
\end{tabular}


Table 15

Bivariate and Partial Correlations of the Predictors with Forward Priming Using the Subset of Related Word Pairs $(N=21)$

\begin{tabular}{lccc}
\hline Predictor & $\begin{array}{c}\text { Correlation between each } \\
\text { predictor and forward } \\
\text { priming }\end{array}$ & $\begin{array}{c}\text { Correlation between each } \\
\text { predictor and forward priming } \\
\text { controlling for other predictor }\end{array}$ \\
\hline Target frequency & .15 & Short SOA & .07 \\
Forward association & -.09 & -.12 \\
Semantic relatedness & -.19 & -.14 \\
\hline Larget frequency & & Long SOA & \\
Forward association & -.06 & -.20 \\
Semantic relatedness & -.06 & -.08 \\
\hline
\end{tabular}


Table 16

Summary of the Multiple Regression Analysis For Variables Predicting the Magnitude of Forward Priming Using the Subset of Related Word Pairs $(N=21)$

\begin{tabular}{lccccc}
\hline & $B$ & $S E B$ & $\beta$ & $R$ & $R^{2}$ \\
\hline & & & Short SOA & \\
Target frequency & 8.20 & 27.61 & .08 & .15 & .02 \\
Forward association & -47.49 & 97.02 & -.12 & .09 & .01 \\
Semantic Relatedness & -37.85 & 65.47 & -.16 & .19 & .04 \\
All & & & & .23 & .05 \\
\hline & & & Long SOA & & \\
Target frequency & -25.13 & 29.14 &.-23 & .06 & .003 \\
Forward association & -35.77 & 102.40 & -.35 & .06 & .004 \\
Semantic Relatedness & -92.95 & 69.10 & -.36 & .24 & .06 \\
All & & & & & .10 \\
\hline
\end{tabular}


Table 17

Bivariate and Partial Correlations of the Predictors with Backward Priming Using the Subset of Related Word Pairs $(N=21)$

\begin{tabular}{lccc}
\hline Predictor & $\begin{array}{c}\text { Correlation between each } \\
\text { predictor and backward } \\
\text { priming }\end{array}$ & $\begin{array}{c}\text { Correlation between each } \\
\text { predictor and backward priming } \\
\text { controlling for other predictor }\end{array}$ \\
\hline Target frequency & $-.50^{*}$ & Short SOA \\
Forward association & $.53^{*}$ & -.38 \\
Semantic relatedness & -.12 & .42 \\
& & -.07 \\
Target frequency & -.26 & Long SOA & -.19 \\
Forward association & .24 & .14 \\
Semantic relatedness & -.23 & -.21 \\
\hline
\end{tabular}

${ }^{*} p<.05$ 
Table 18

Summary of the Multiple Regression Analysis for Variables Predicting the Magnitude of Backward Priming Using the Subset of Related Word Pairs $(N=21)$

\begin{tabular}{lccccc}
\hline & $B$ & $S E B$ & $\beta$ & $R$ & $R^{2}$ \\
\hline Target frequency & -52.28 & 31.47 & -.34 & .50 & $.25^{*}$ \\
Forward association & 300.75 & 159.44 & .39 & .53 & $.28^{*}$ \\
Semantic Relatedness & -23.84 & 86.55 & -.05 & .12 & .01 \\
All & & & & .62 & $.38^{*}$ \\
\hline & & & Long SOA & & \\
Target frequency & -11.50 & 14.51 & -.19 & .26 & .07 \\
Forward association & 43.02 & 73.52 & .14 & .24 & .06 \\
Semantic Relatedness & -35.75 & 39.91 & -.20 & .23 & .05 \\
All & & & & & .13 \\
\hline${ }^{*} p<.05$. & & & & &
\end{tabular}


Table 19

Mean Error Scores (Root MSE) And Priming

Results For The Neural Network

\begin{tabular}{lcc} 
& \multicolumn{2}{c}{ Priming Direction } \\
\cline { 2 - 3 } Prime Type & Forward & Backward \\
\hline & \multicolumn{2}{c}{ Short SOA } \\
Related & $.92(0.47)$ & $0.98(0.58)$ \\
Unrelated & $1.15(0.49)$ & $1.13(0.54)$ \\
Priming & .23 & .15 \\
\hline & \multicolumn{2}{c}{ Long SOA } \\
Related & $1.02(0.50)$ & $.99(0.57)$ \\
Unrelated & $1.11(0.50)$ & $1.07(0.55)$ \\
Priming & .09 & .08 \\
\hline
\end{tabular}

Note. Values enclosed in parentheses represent s standard deviation values. 
Table 20

Analysis of Variance for Neural Network Simulation (Participant) Mean Root MSE Values

\begin{tabular}{lcccc}
\hline \multicolumn{1}{c}{ Source } & $d f$ & $F$ & $\eta^{2}$ & $p$ \\
\hline & & \multicolumn{2}{c}{ Within subjects } \\
SOA (S) & 1 & 0.000 & .000 & .99 \\
Direction (D) & 1 & 0.27 & .07 & .61 \\
Prime (P) & 1 & $1895.04^{* *}$ & .99 & .000 \\
$\mathrm{~S} \times \mathrm{D}$ & 1 & $15.28^{*}$ & .35 & .001 \\
$\mathrm{~S} \times \mathrm{P}$ & 1 & $436.61^{* *}$ & .99 & .000 \\
$\mathrm{D} \times \mathrm{P}$ & 1 & $47.30^{* *}$ & .62 & .000 \\
$\mathrm{~S} \times \mathrm{D} \times \mathrm{P}$ & 1 & $59.60^{* *}$ & .67 & .000 \\
$\mathrm{~S}$ within-group & & & & \\
$\quad$ error & 29 & $(0.01)$ & & \\
\hline
\end{tabular}

Note. Values enclosed within parentheses represent mean square errors. ${ }^{*} p<.01 .{ }^{* *} p<.001$. 
Table 21

Analysis of Variance for Neural Network Word Item Mean Root MSE Values

\begin{tabular}{lcccc}
\hline \multicolumn{1}{c}{ Source } & $d f$ & $F$ & $\eta^{2}$ & $p$ \\
\hline & & \multicolumn{2}{c}{ Within subjects } \\
SOA (S) & 1 & 0.000 & .000 & .99 \\
Direction (D) & 1 & 0.002 & .000 & .97 \\
Prime (P) & 1 & $5.59^{*}$ & .20 & .03 \\
S $\times$ D & 1 & 2.87 & .11 & .10 \\
S $\times$ P & 1 & $11.10^{* *}$ & .33 & .003 \\
D $\times$ P & 1 & 0.21 & .01 & .65 \\
S $\times$ D $\times$ P & 1 & 1.39 & .06 & .25 \\
D within-group & & & & \\
$\quad$ error & 23 & $(0.51)$ & \\
\hline$N$ Not Values & & & \\
\hline
\end{tabular}

Note. Values enclosed within parentheses represent mean square errors.

${ }^{*} p<.05 .{ }^{* *} p<.001$. 
Table 22

Bivariate and Partial Correlations of the Predictors with Forward Priming in the Neural Network $(N=24)$

\begin{tabular}{lccc}
\hline & $\begin{array}{c}\text { Correlation between each } \\
\text { predictor and forward } \\
\text { priming }\end{array}$ & $\begin{array}{c}\text { Correlation between each } \\
\text { predictor and forward priming } \\
\text { controlling for other predictor }\end{array}$ \\
\hline Target frequency & $.47^{*}$ & Short SOA & $.47^{*}$ \\
Semantic relatedness & -.06 & -.07 \\
\hline & & Long SOA & $.50^{*}$ \\
Target frequency & $.50^{*}$ & .02 \\
Semantic relatedness & .02 & \\
\hline${ }^{*} p<.05$. & &
\end{tabular}


Table 23

Summary of the Multiple Regression Analysis for Lexical Frequency of the Target Word and Forward Association Predicting Magnitude of Forward Priming in the Neural Network $(N=24)$

\begin{tabular}{lccccc}
\hline & $B$ & $S E B$ & $\beta$ & $R$ & $R^{2}$ \\
\hline & & & Short SOA & \\
Target frequency & 0.46 & 0.19 & $.47^{*}$ & .56 & $.31^{* *}$ \\
Semantic relatedness & -0.06 & 0.19 & -.06 & .07 & .01 \\
All Predictors & & & & .47 & .22 \\
\hline & & & Long SOA & & \\
Target frequency & 0.58 & 0.22 & $0.50^{*}$ & .64 & $.41^{* *}$ \\
Semantic relatedness & 0.02 & 0.22 & 0.02 & .22 & .05 \\
All Predictors & & & & .50 & .25 \\
${ }^{*} p<.05 .{ }^{* *} p<.01$. & & & & &
\end{tabular}


Table 24

Bivariate and Partial Correlations of the Predictors with Backward Priming in the Neural Network $(N=24)$

\begin{tabular}{|c|c|c|}
\hline Predictor & $\begin{array}{c}\text { Correlation between each } \\
\text { predictor and backward } \\
\text { priming }\end{array}$ & $\begin{array}{l}\text { Correlation between each } \\
\text { predictor and backward priming } \\
\text { controlling for other predictor }\end{array}$ \\
\hline & \multicolumn{2}{|c|}{ Short SOA } \\
\hline Target frequency & -.13 & -.14 \\
\hline \multirow[t]{2}{*}{ Semantic relatedness } & .28 & .28 \\
\hline & \multicolumn{2}{|c|}{ Long SOA } \\
\hline Target frequency & -.25 & -.25 \\
\hline Semantic relatedness & $-.47^{*}$ & $-.47^{*}$ \\
\hline
\end{tabular}


Table 25

Summary of the Multiple Regression Analysis for Lexical Frequency of the Target Word and Forward Association Predicting Magnitude of Backward Priming in the Neural Network $(N=24)$

\begin{tabular}{lccccc}
\hline & $B$ & $S E B$ & $\beta$ & $R$ & $R^{2}$ \\
\hline & & & Short SOA & \\
Target frequency & -0.03 & 0.05 & -.13 & .13 & .02 \\
Semantic relatedness & 0.09 & 0.05 & .38 & .28 & .08 \\
All Predictors & & & & .41 & .16 \\
\hline & & & Long SOA &. & \\
Target frequency & -0.04 & 0.03 & -.25 & .25 & .06 \\
Semantic relatedness & 0.02 & 0.03 & .16 & .47 & $.22 *$ \\
All Predictors & & & & .30 & .09 \\
${ }^{*} p<.05$. & & & & &
\end{tabular}


Figure 1

Architecture of the simple recurrent neural network used in the study. Solid arrow lines signify fully connected weight connections and the broken arrow line signifies one-to-one weight connections.

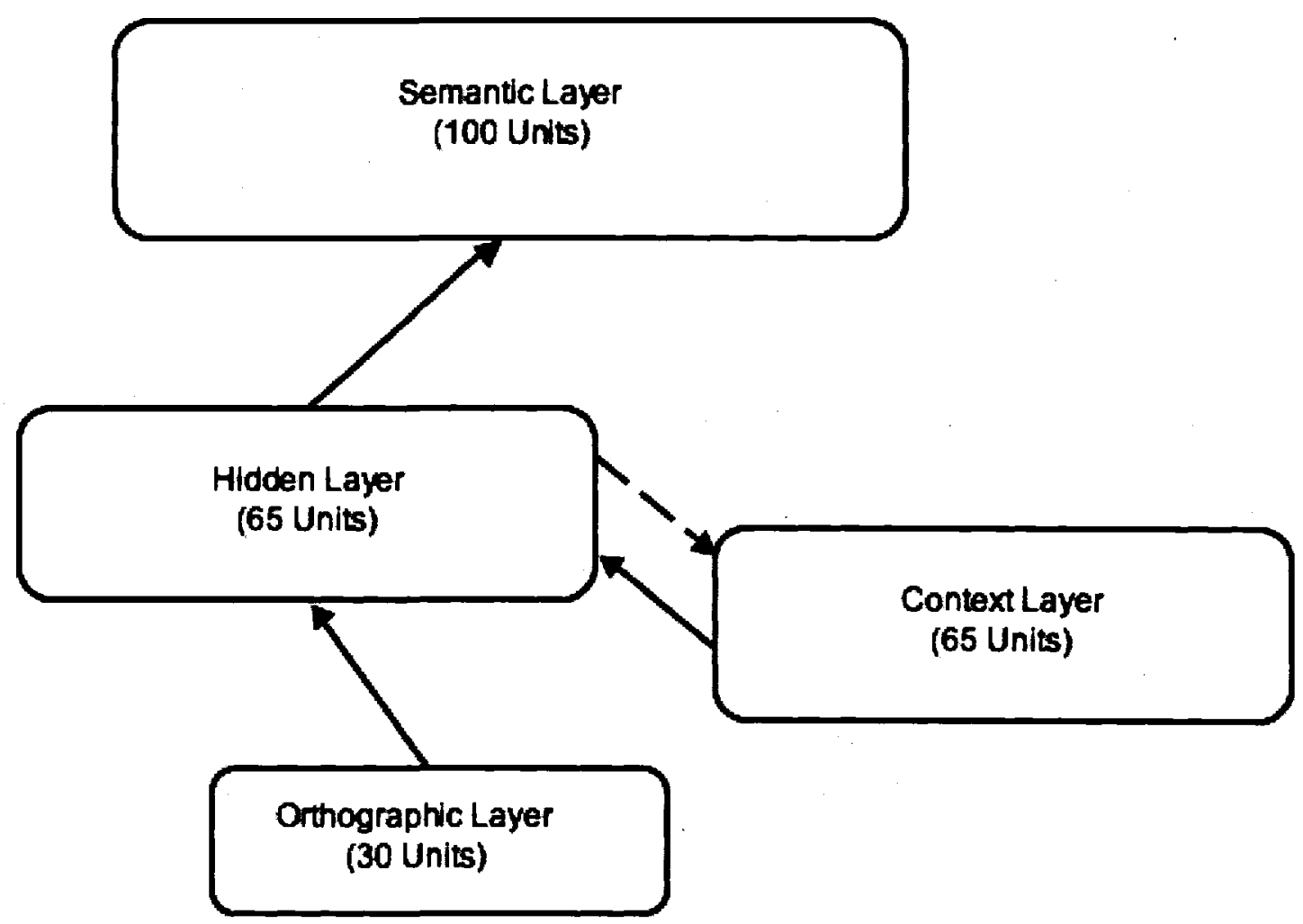


Figure 2

Cluster analysis for hidden activation states of related prime (Prm) and target (Tar) words when presented in isolation and distinguished by semantic relatedness (High vs. Low). The * signifies a related word pair properly paired in the same leaf cluster.

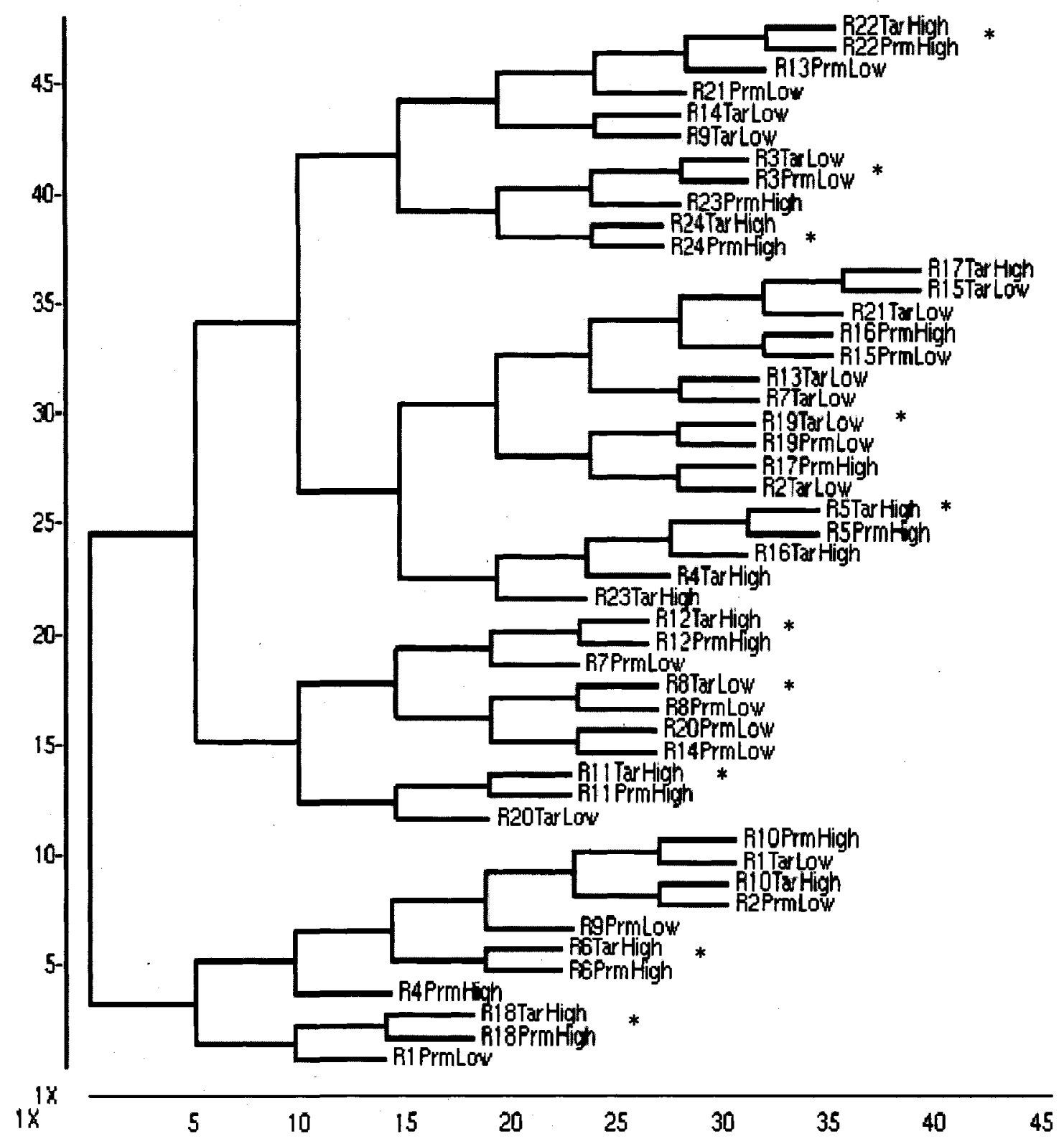


Figure 3

Cluster analysis for hidden activation states for unrelated prime (Prm) and target (Tar) words when presented in isolation distinguished by semantic relatedness (High vs. Low).

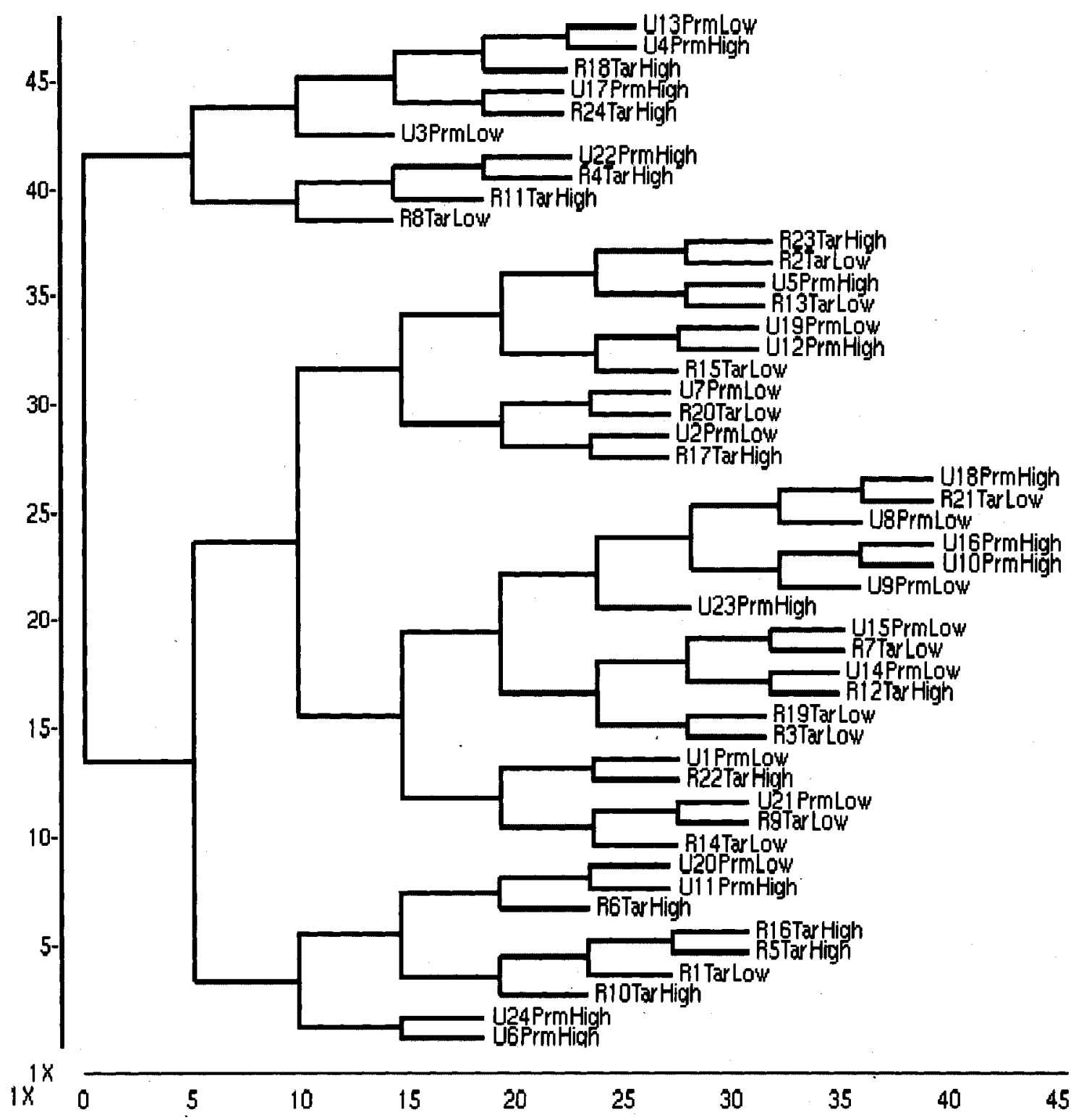


Figure 4

Cluster analysis for context layer activation states for related prime (Prm) and target (Tar) words when presented together in the backward direction distinguished by semantic relatedness (High vs. Low). The * signifies a word that was not properly paired with its associated word in the same leaf cluster.

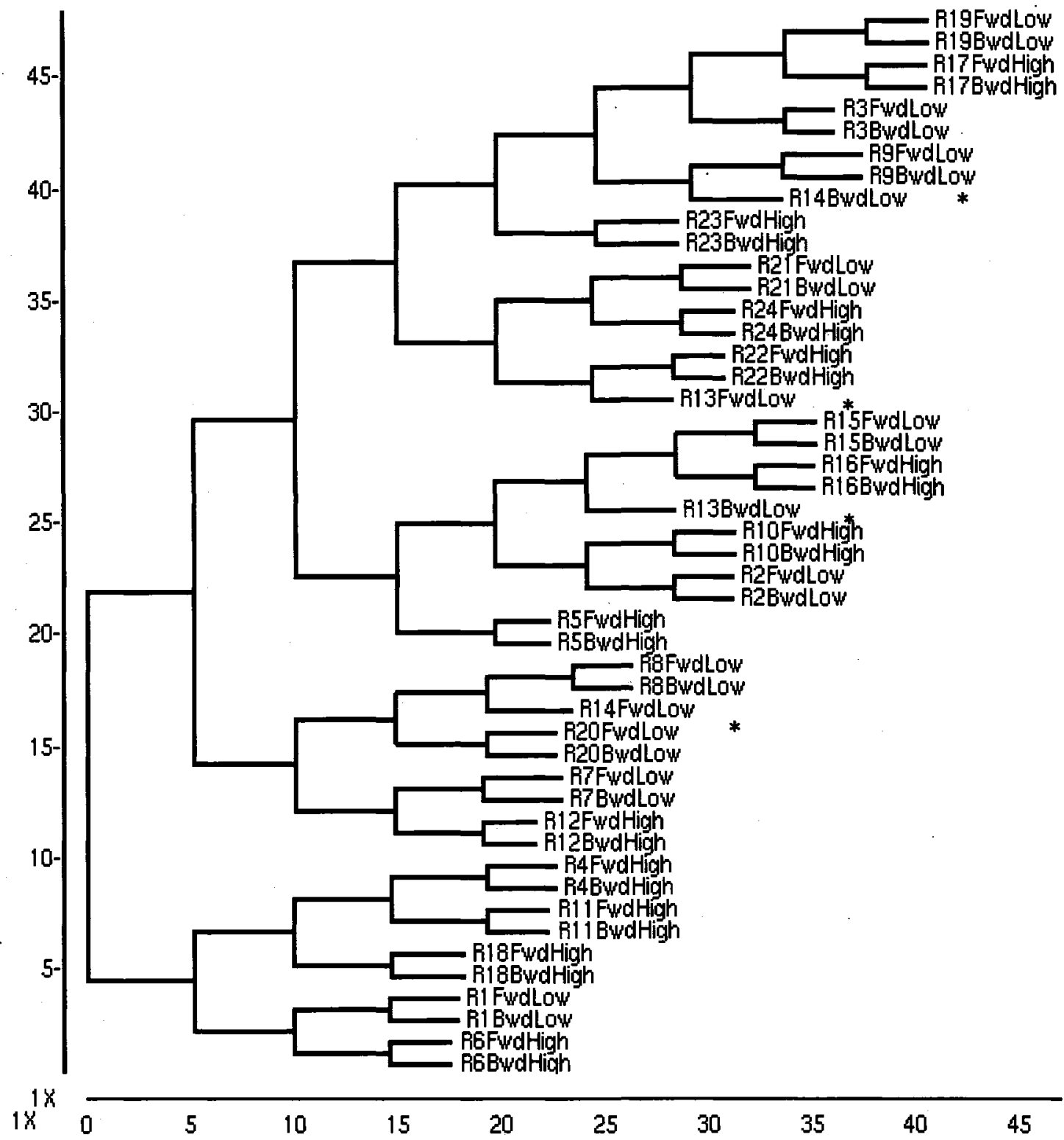

